\title{
An Extended Cosine Generalized Family of Distributions for Reliability Modeling: Characteristics and Applications with Simulation Study
}

\author{
Zafar Mahmood $\mathbb{D},{ }^{1}$ Taghreed M Jawa $\mathbb{D}^{2},{ }^{2}$ Neveen Sayed-Ahmed $\mathbb{D}^{2},{ }^{2}$ E M Khalil $\mathbb{D},{ }^{2}$ \\ Abdisalam Hassan Muse $\oplus^{3},{ }^{3}$ and Ahlam H. Tolba $\oplus^{4}$ \\ ${ }^{1}$ Department of Statistics, Government Associate College, Khairpur Tamewali, Bahawalpur, Pakistan \\ ${ }^{2}$ Department of Mathematics, College of Science, P.O. Box 11099, Taif University, Taif 21944, Saudi Arabia \\ ${ }^{3}$ Department of Mathematics (Statistics Option) Programme, Pan African University, \\ Institute of Basic Science, Technology and Innovation (PAUSTI), Nairobi 6200-00200, Kenya \\ ${ }^{4}$ Department of Mathematics, Faculty of Science, Mansoura University, Mansoura 35516, Egypt
}

Correspondence should be addressed to Abdisalam Hassan Muse; abdisalam.hassan@amoud.edu.so

Received 24 October 2021; Revised 9 January 2022; Accepted 24 January 2022; Published 27 February 2022

Academic Editor: Dost Muhammad Khan

Copyright $\odot 2022$ Zafar Mahmood et al. This is an open access article distributed under the Creative Commons Attribution License, which permits unrestricted use, distribution, and reproduction in any medium, provided the original work is properly cited.

\begin{abstract}
An extension of the cosine generalized family is presented in this paper by using the cosine trigonometric function and method of parameter induction concurrently. Prominent characteristics of the proposed family along with useful results are extracted. Moreover, two new subfamilies and several special models are also deduced. A four-parameter model called an Extended Cosine Weibull $(E C W)$ with its mathematical properties is studied deeply. Graphical study reveals that the new model adopts right- and left-skewed, symmetrical, and reversed-J density shapes, while all possible monotone and nonmonotone shapes are exhibited by the hazard rate function. The maximum likelihood technique is exercised for parametric estimation, while estimation performance is accessed via Monte Carlo simulation study graphically and numerically. The superiority of the presented model over several outstanding and competing models is confirmed via three reliability and survival dataset applications.
\end{abstract}

\section{Introduction with Motivations}

This twenty-first century started with setting up and broadening new graphing and analytical instruments for current modern statistics. One novel development is the introduction of extended and generalized distributions utilizing classical one and further certified availing real and lifetime data accessible from easy to complex phenomenon. In this way, improved estimation of model parameters and model goodness of fit is achieved that is effectively applicable in environmental sciences, biological sciences, industry, agricultural biotechnology, engineering, economics, forestry, and many others.
An immense research work about the theory and applications of statistical distributions exists in the literature. The first reason is the thrust of statisticians to develop novel and flexible models possessing significant mathematical and graphical characteristics. The struggle, challenging, and endless work of statisticians bore fruit, and many modified, extended, and generalized families of distributions are introduced; for more information, see [2-4]. The literature review explores the second reason that simple and nongeneralized models provide an inadequate fit compared to extended and generalized models, especially in real-life situations. The third and very important fact is that the data behave in a more complex way than what is commonly expected in many disciplines. 
For a brief review, the reader is referred to the memorable contribution [5] about the transformed- (T-) transformer (X) mechanism of developing new families of statistical distributions, extended work [6] regarding the McDonald-G family, and pivotal work on exponentiated generalized families [7]. Table 1 presents a review of trigonometric function-based models chronologically.

A concise summary of the cosine-G family is as follows: let $G(x)$ (cumulative distribution function (cdf) of baseline), then new cdf, probability density function (pdf), and hazard rate function (hrf), respectively, be $F(x)=1-\cos (\pi / 2 G(x))$, $f(x)=\pi / 2 g(x) \sin (\pi / 2 G(x))$, and $h(x)=\pi / 2 g(x) \tan (\pi /$ $2 G(x)), x \in \mathbb{R}$. Lehmann (1953) presented Lehmann alternative-1 (shortly LA-1) method, which is a famous method of constructing exponentiated families (EF) of distributions and is also defined by Gupta et al. (1998) as $F(x)=(G(x))^{\alpha}$.

Moreover, the key aspects that become foundations for this study are as follows: (1) A large variety of nontrigonometric $\mathrm{G}$ families are added in the statistical literature, only utilizing the algebraic generalizers/generators of distributions $W[G(x)]$ while disregarding trigonometric ones (trigonometric generalizers). (2) The trend in modeling directional and proportional data prompted applied researchers to construct trigonometric function-based statistical models that can be more efficient and effective. (3) The algebraic and trigonometric functions mixed generalizers are not developed and consumed yet.

Influenced by foundation points and the produced results regarding accuracy, flexibility, and goodness of fit (GoF), the key motivations for introducing an extended cosine-G family are as follows:

(i) Pursuing the enthusiasm of the cosine-G family and LA-1 mechanism of constructing new $G$ families simultaneously, we develop an extended version of the cosine-G family.

(ii) The proposed family has a lot of advantages, including simplicity, explicit expressions for central functions, free from nonidentifiability issue, and overparametrization.

(iii) The extended cdf can boost flexibility, accuracy, and GoF due to the injection of shape parameters, thus resulting in new flexible and efficient models. (iv) Adopting the suggested extension, any G class or model can be readily reverted in a subsequent version, which will be trigonometric and doubly exponentiated.

(v) The existing literature attests that nongeneralized, nonexponentiated, and nonextended models provide inadequate GoF.

(vi) The produced extended models are efficient in modeling with monotonic and nonmonotonic hazard functions.

Moreover, recent remarkable $\mathrm{G}$ families are presented in Table 2 in connection with the adopted methodology.

The paper layout is as follows: after the introduction with motivations in Section 1, an extended cosine-G family with general mathematical properties is presented in Section 2. A special member $(E C W)$ is briefly studied in Section 3 . Parametric estimation, simulation study, and applications on two reliability datasets are performed by taking $(E C W)$ as a statistical model in Section 4. Concluding remarks are stated in Section 5.

\section{The Proposed Family and Significant Characteristics}

The construction procedure of the proposed family with significant characteristics is presented in this section.

2.1. Construction Procedure. The idea behind the proposed family is to apply the process of exponentiated families (EF) to the former cosine-G family. Indeed, we can write

$$
F(x)=\left[1-\cos \left(\frac{\pi}{2} H(x)\right)\right]^{\beta},
$$

where $H(x)$ denotes the exponentiated (generalized using LA-1) cdf of $(G(x))^{\alpha}$ and again $F(x)=[K(x)]^{\beta}$, where $K(x)=[1-\cos (\pi / 2 H(x))]$.

2.2. Main Functions. The cdf and pdf of "an extended cosine-G” (shortly an ext cos-G), respectively, are

$$
\begin{aligned}
& F(x)=\left[1-\cos \left(\frac{\pi}{2} G(x)^{\alpha}\right)\right]^{\beta}, \quad x \in \mathbb{R}, \\
& f(x)=\frac{\pi}{2} \alpha \beta g(x) G(x)^{\alpha-1} \sin \left(\frac{\pi}{2} G(x)^{\alpha}\right)\left[1-\cos \left(\frac{\pi}{2} G(x)^{\alpha}\right)\right]^{\beta-1} .
\end{aligned}
$$


TABLe 1: Chronological review on trigonometric functions based on $G$ families and distributions.

\begin{tabular}{lcc}
\hline S. no. & Introducer(s) (year) & Model based on trigonometric function \\
\hline 1 & Raab and Green (1961) & Derived a cosine distribution [8] \\
2 & Nadarajah and Kotz (2006) & Derived the beta trigonometric distribution [9] \\
3 & Chakraborty et al. (2012) & Explored the sin-skew logistic distribution [10] \\
4 & Souza (2015) & Explored the cosine sine (CS) distribution [11] \\
5 & Chesneau et al. (2019) & Suggested new trigonometric classes of probabilistic distributions [1] \\
6 & Souza et al. (2019) & Derived mathematical characteristics for cos-G class [12] \\
7 & Mahmood et al. (2019) & Presented a new sine-G family of distributions [13] \\
8 & Chesneau et al. (2020) & Proposed the cosine geometric distribution [14] \\
9 & Chesneau et al. (2020) & Deduced sine Kumaraswamy-G family of distribution [15] \\
10 & Al-Babtain et al. (2020) & Introduced sine Topp-Leone-G family [16] \\
11 & Ali (2021) & Worked on sine power Lomax model [17] \\
12 & Alkhairy et al. (2021) & Introduced the arctangent-X family of distributions [18] \\
13 & Nagarjuna et al. (2021) & Worked on the accuracy of the sine power Lomax model [19] \\
14 & Nagarjuna et al. (2021) & Presented Kumaraswamy generalized power Lomax distribution [20] \\
15 & Ahmed et al. (2021) & Presented generalized power Akshaya distribution with applications [21] \\
16 & Muse et al. (2021) & Introduced the Tan log-logistic distribution [22] \\
17 & Almetwally (2021) & Presented odd Weibull inverse Topp-Leone distribution [23] \\
18 & Hassan et al. (2021) & Presented Kumaraswamy inverted Topp-Leone distribution [24] \\
19 & Almetwally et al. (2021) & Presented Marshall-Olkin alpha Power-X distribution [25-27] \\
20 & Almongy et al. (2021) & Presented extended odd Weibull Rayleigh [28] \\
21 & Al-Babtain et al. (2021) & Presented the flexible burr-XG family [29]
\end{tabular}

2.3. Subfamilies and New Members of an Ext Cos-G Family. This proposed family possesses three $G$ families (two new and one existing) as subfamilies, which are presented in Table 3 and are deduced just by specifying the parametric values.

In Table 4, eight new members are introduced, for example, utilizing the famous statistical distributions on all possible intervals.
2.4. Useful Reliability Functions. The hazard rate function (hrf) $h(x)$, survival function (sf) $S(x)$, reversed hazard rate function (rhrf) $r(x)$, cumulative hazard rate function (chrf) $H(x)$, mills' ratio $m(x)$, conditional reliability function $\bar{G}(G(x), \alpha, \beta \mid t)$, and elasticity $e(x)$ are, respectively, given under

$$
\begin{aligned}
h(x) & =\frac{\pi / 2 \alpha \beta g(x) G(x)^{\alpha-1} \sin \left(\pi / 2 G(x)^{\alpha}\right)\left[1-\cos \left(\pi / 2 G(x)^{\alpha}\right)\right]^{\beta-1}}{1-\left[1-\cos \left(\pi / 2 G(x)^{\alpha}\right)\right]^{\beta}}, \\
S(x) & =1-\left[1-\cos \left(\frac{\pi}{2} G(x)^{\alpha}\right)\right]^{\beta}, \\
r(x) & =\frac{\pi}{2} \alpha \beta g(x) G(x)^{\alpha-1} \sin \left(\frac{\pi}{2} G(x)^{\alpha}\right)\left[1-\cos \left(\frac{\pi}{2} G(x)^{\alpha}\right)\right]^{-1}, \\
H(x) & =-\ln \left[1-\left[1-\cos \left(\frac{\pi}{2} G(x)^{\alpha}\right)\right]^{\beta}\right], \\
m(x) & =\frac{1-\left[1-\cos \left(\pi / 2 G(x)^{\alpha}\right)\right]^{\beta}}{\pi / 2 \alpha \beta g(x) G(x)^{\alpha-1} \sin \left(\pi / 2 G(x)^{\alpha}\right)\left[1-\cos \left(\pi / 2 G(x)^{\alpha}\right)\right]^{\beta-1}}, \\
\bar{G}(G(x), \alpha, \beta \mid t) & =\frac{1-\left[1-\cos \left(\pi / 2 G(x+t)^{\alpha}\right)\right]^{\beta}}{1-\left[1-\cos \left(\pi / 2 G(t)^{\alpha}\right)\right]^{\beta}}, \\
e(x) & =\frac{\partial}{\partial(\ln G(x))}\left[\ln \left(1-\cos \left(\frac{\pi}{2} G(x)^{\alpha}\right)\right)^{\beta}\right] .
\end{aligned}
$$


TABle 2: Some recent G families of distributions.

\begin{tabular}{lcc}
\hline Introducer(s) & Proposed G family & Adopted methodology \\
\hline Hamdani et al. (2021) & Type I quasi-Lambert family & Lambert function [30] \\
Altun et al. (2021) & Additive odd-G family & Additive model structure [31] \\
Aljarrah et al. (2021) & Generator of distributions & Quantile function [32] \\
Salahuddin et al. (2021) & New family & Better GoF and flexibility [33] \\
Korkamaz et al. (2021) & New class & Hjorth's IDB generator [34] \\
Cordeiro et al. (2020) & XGamma family & Parameter induction [35] \\
Korkamaz et al. (2019) & TL Gen.Odd log-Logistic family & Odd generator [36] \\
Alzaatreh et al. (2019) & G-classes & T-X methodology [5] \\
Bhatti et al. (2019) & On burr III MO family & Hazard rate function [37] \\
Korkamaz et al. (2018) & New G-classes & Extended distribution [35] \\
Alizadeh et al. (2019) & Another odd log-logistic logarithmic-G & Odd generator [38] \\
Algarni et al. (2021) & Type I half logistic Burr-XG & Extended T-X methodology [39] \\
\hline
\end{tabular}

Table 3: Subfamilies of an ext cos-G family.

\begin{tabular}{lccccc}
\hline S. no. & $\alpha$ & $\beta$ & Subfamilies of distributions & Comments \\
1 & 1 & - & Generalized cosine-G family (using LA-1 technique) & New \\
2 & - & 1 & Cosine exponentiated-G family & Existing \\
3 & 1 & 1 & Cosine-G family [1] & \\
\hline
\end{tabular}

TABLe 4: Members of an ext cos-G family and their cdfs.

\begin{tabular}{lccc}
\hline Baseline model & Support & New cdf $F(x)$ & Parameters \\
\hline Topp-Leone & $(0,1)$ & {$\left[1-\cos \left(\pi / 2(x(2-x))^{\alpha}\right)\right]^{\beta}$} & $(\alpha, \beta)$ \\
Uniform & $(0, \theta)$ & {$\left[1-\cos \left(\pi / 2(x / \theta)^{\alpha}\right)\right]^{\beta}$} & $(\theta, \alpha, \beta)$ \\
Exponential & $(0, \infty)$ & {$\left[1-\cos \left(\pi / 2\left(1-e^{-\lambda x)^{\alpha}}\right)\right]^{\beta}\right.$} & $(\lambda, \alpha, \beta)$ \\
Weibull & $(0, \infty)$ & {$\left[1-\cos \left(\pi / 2\left(1-e^{-\lambda x^{\sigma}}\right){ }^{\alpha}\right)\right]^{\beta}$} & $(\sigma, \lambda, \alpha, \beta)$ \\
Burr XII & $(0, \infty)$ & {$\left[1-\cos \left(\pi / 2\left(1-\left(1+(x / s)^{c}\right)^{-k}\right)^{\alpha}\right)\right]^{\beta}$} & $(s, c, k, \alpha, \beta)$ \\
Logistic & $\mathbb{R}$ & {$\left[1-\cos \left(\pi / 2\left(\left(1+e^{-(x-\mu) / s}\right)^{-1}\right)^{\alpha}\right)\right]^{\beta}$} & $(s, \mu, \alpha, \beta)$ \\
Gumbel & $\mathbb{R}$ & {$\left[1-\cos \left(\pi / 2\left(\mathrm{e}^{\left(-e^{-(x-\mu) / \sigma}\right)}\right)^{\alpha}\right)\right]^{\beta}$} & $(\sigma, \mu, \alpha, \beta)$ \\
Normal & $\mathbb{R}$ & {$\left[1-\cos (\pi / 2(\Phi((x-\mu) / \sigma)))^{\alpha}\right]^{\beta}$} & $(\sigma, \mu, \alpha, \beta)$ \\
\hline
\end{tabular}

2.5. Quantile Function and Quantile Density Function. An additional property of $X$ is quantile function (qf), which is derived from the direct inverter (2) as

$$
Q_{G}(v)=G^{-1}\left(\frac{2}{\pi} \cos ^{-1}\left(1-u^{1 / \beta}\right)^{1 / \alpha}\right),
$$

where $u$ has a uniform $U(0,1)$ distribution, while the quantile density function (qdf) is

$$
q d f=\frac{2(u)^{1-\beta / \beta}\left(2 / \pi \cos ^{-1}\left(1-u^{1 / \beta}\right)^{1-\alpha / \alpha}\right)}{\pi \alpha \beta\left(1-\left(1-u^{1 / \beta}\right)^{2}\right)} .
$$

2.6. Analytical Points of the Density and Hazard Functions. The solution $x_{0}$ of the nonlinear equation $f^{\prime}\left(x_{0}\right)=0$ presents the analytical points of density, which are

$$
\begin{aligned}
& \frac{1}{2} \pi \alpha \beta G\left(x_{0}\right)^{\alpha-1} g^{\prime}\left(x_{0}\right) \sin \left(\frac{1}{2} \pi G\left(x_{0}\right)^{\alpha}\right)\left[1-\cos \left(\frac{1}{2} \pi G\left(x_{0}\right)^{\alpha}\right)\right]^{\beta-1} \\
& \quad+\frac{1}{4} \pi^{2} \alpha^{2} \beta G\left(x_{0}\right)^{2 \alpha-2} g\left(x_{0}\right)^{2} \cos \left(\frac{1}{2} \pi G\left(x_{0}\right)^{\alpha}\right)\left[1-\cos \left(\frac{1}{2} \pi G\left(x_{0}\right)^{\alpha}\right)\right]^{\beta-1} \\
& \quad+\frac{1}{4} \pi^{2} \alpha^{2}(\beta-1) \beta G\left(x_{0}\right)^{2 \alpha-2} g\left(x_{0}\right)^{2} \sin ^{2}\left(\frac{1}{2} \pi G\left(x_{0}\right)^{\alpha}\right)\left[1-\cos \left(\frac{1}{2} \pi G\left(x_{0}\right)^{\alpha}\right)\right]^{\beta-2} \\
& +\frac{1}{2} \pi(\alpha-1) \alpha \beta G\left(x_{0}\right)^{\alpha-2} g\left(x_{0}\right)^{2} \sin \left(\frac{1}{2} \pi G\left(x_{0}\right)^{\alpha}\right)\left[1-\cos \left(\frac{1}{2} \pi G\left(x_{0}\right)^{\alpha}\right)\right]^{\beta-1}=0 .
\end{aligned}
$$


Similarly, the analytical points of the hazard function are obtained by the solution $x_{*}$ of $h^{\prime}\left(x_{*}\right)=0$.

$$
\begin{aligned}
& \frac{1}{2} \pi \alpha \beta G\left(x_{*}\right)^{\alpha-1} g^{\prime}\left(x_{*}\right) \sin \left(\frac{1}{2} \pi G\left(x_{*}\right)^{\alpha}\right)\left[1-\cos \left(\frac{1}{2} \pi G\left(x_{*}\right)^{\alpha}\right)\right]^{\beta-1} \\
& +\frac{1}{4} \pi^{2} \alpha^{2} \beta G\left(x_{*}\right)^{2 \alpha-2} g\left(x_{*}\right)^{2} \cos \left(\frac{1}{2} \pi G\left(x_{*}\right)^{\alpha}\right)\left[1-\cos \left(\frac{1}{2} \pi G\left(x_{*}\right)^{\alpha}\right)\right]^{\beta-1} \\
& +\frac{1}{4} \pi^{2} \alpha^{2}(\beta-1) \beta G\left(x_{*}\right)^{2 \alpha-2} g(x)^{2} \sin ^{2}\left(\frac{1}{2} \pi G\left(x_{*}\right)^{\alpha}\right)\left[1-\cos \left(\frac{1}{2} \pi G\left(x_{*}\right)^{\alpha}\right)\right]^{\beta-2} \\
& +\frac{1}{2} \pi(\alpha-1) \alpha \beta G\left(x_{*}\right)^{\alpha-2} g\left(x_{*}\right)^{2} \sin \left(\frac{1}{2} \pi G\left(x_{*}\right)^{\alpha}\right)\left[1-\cos \left(\frac{1}{2} \pi G\left(x_{*}\right)^{\alpha}\right)\right]^{\beta-1} \\
& \quad-\frac{1}{2} \pi \alpha \beta G\left(x_{*}\right)^{\alpha-1} g\left(x_{*}\right) \sin \left(\frac{1}{2} \pi G\left(x_{*}\right)^{\alpha}\right)\left[1-\cos \left(\frac{1}{2} \pi G\left(x_{*}\right)^{\alpha}\right)\right]^{\beta-1}=0 .
\end{aligned}
$$

2.7. Useful Expansions. It is a good idea to have a distribution function power series representation in terms of the baseline distribution's positive integer powers. Using the series expansions for the cosine function, $F(x)$ can be expressed as a linear sum of power cdfs. For example, $\theta$ is an integer then $[G(x)]^{\theta}$. Thus, the cdf (2) allows the expansion

$$
F(x)=\left[1-\cos \left(\frac{\pi}{2} G(x)^{\alpha}\right)\right]^{\beta}=\sum_{i=0}^{\infty}(-1)^{i}\left(\begin{array}{c}
\beta \\
i
\end{array}\right)\left[\cos \left(\frac{\pi}{2} G(x)^{\alpha}\right)\right]^{i}
$$

Via Mathematica 11.0, $[\cos (x)]^{i}=\sum_{j=0}^{\infty} b_{j}(i)[x]^{2 j}$, where $b_{0}(i)=1, b_{1}(i)=-i / 2, b_{2}(i)=i(3 i-2) / 24$, and so on. Thus,

$$
\left[\cos \frac{\pi}{2} G(x)^{\alpha}\right]^{i}=\sum_{k=0}^{\infty} b_{k}(i)\left(\frac{\pi}{2} G(x)^{\alpha}\right)^{2 k} .
$$

Finally, the linear representation for cdf is

$$
F(x)=\sum_{k=0}^{\infty} W_{(i, k)} H_{(2 k \alpha)}(x)
$$

where $H_{(2 k \alpha)}(x)$ is the exponentiated cdf with $(2 k \alpha)$ as power parameter and

$$
W_{(i, k)}=\sum_{i=0}^{\infty}(-1)^{(i)} b_{k}(i)\left(\begin{array}{c}
\beta \\
i
\end{array}\right)\left(\frac{\pi}{2}\right)^{2 k} \text {. }
$$

Similarly, the expansion for (3) is

$$
f(x)=\sum_{k=0}^{\infty} v_{(i, k)} h_{(2 k \alpha)}(x)
$$

where $h_{(2 k \alpha)}(x)$ is the exponentiated density with $(2 k \alpha)$ as power parameter and

$$
\begin{aligned}
& v_{(i, k)}=\sum_{i=0}^{\infty}(-1)^{(i)} b_{k}(i)\left(\begin{array}{l}
\beta \\
i
\end{array}\right)\left(\frac{\pi}{2}\right)^{2 k}, \\
& b_{0}(i)=1 \\
& b_{1}(i)=-\frac{i}{2}, \\
& b_{2}(i)=i \frac{(3 i-2)}{24}, \\
& \text { etc. }
\end{aligned}
$$

And

$$
h_{(\alpha(2 k))}(x)=2 k \alpha g(x) G(x)^{2 k \alpha-1} .
$$

Equation (13) proves that the new density is a true linear combination of exp-G densities. Therefore, utilizing those exp-G properties, various properties of the new family can be extracted easily.

2.8. Moments, Incomplete Moments, and Moment Generating Function. The $r$-th moment of $X$ is expressed as $\mu_{r}^{\prime}=\mathbb{E}\left(X^{r}\right)=\int_{0}^{\infty} x^{r} f(x) d x$. By using (13) and assuming that all the sum and integral terms exist, we get 


$$
\mu_{r}^{\prime}=\sum_{k=0}^{\infty} v_{(i, k)} 2 k \alpha \int_{0}^{\infty} x^{r}[G(x)]^{2 k \alpha-1} g(x) d x=\sum_{k=0}^{\infty} v_{(i, k)} \mathbb{E}\left(Y_{k}^{r}\right)
$$

where $Y_{k}$ expresses the exp-G distribution with $(2 k \alpha)$ as power parameter.

The $y$-th incomplete moment of $X$ is

$$
I_{x}(y)=\sum_{k=0}^{\infty} v_{(i, k)} \mathbb{E}\left(I_{k}^{r}\right)
$$

where $I_{k}^{r}(y)=\int_{0}^{y} x^{r} h_{(\alpha(2 k))}(x) d x$.

The corresponding moment generating function (mgf) of $X$ is

$$
M(t)=\sum_{k=0}^{\infty} v_{(i, k)}(2 k \alpha) \int_{0}^{\infty} e^{t x} g(x)[G(x)]^{2 k \alpha-1} d x=\sum_{k=0}^{\infty} v_{(i, k)} \mathbb{E}\left[\mathrm{e}^{t x}\right]
$$

2.9. Probability Weighted Moments (PWMs). The $(r, s)$-th PWM of $X$ (for $r \geq 1, s \geq 0$ ) is properly defined by

$$
\rho_{r, s}=\mathbb{E}\left(X^{r} F(X)^{s}\right)=\int_{0}^{\infty} x^{r} F(x)^{s} f(x) d x
$$

$$
F(x)^{s}=\left[1-\cos \left(\frac{\pi}{2} G(x)^{\alpha}\right)\right]^{s \beta} .
$$

Substituting (3) and (20) into (19) and then applying binomial series expansion, $P W M=\rho_{r, q}$ becomes

(Greenwood, Landwehr, Matalas, and Wallis, 1979). Then,

$$
\rho_{r, q}=\sum_{i=0}^{\infty}(-1)^{i} \frac{\pi}{2} \alpha \beta\left(\begin{array}{c}
\beta(s+1)-1 \\
i
\end{array}\right) \int_{-\infty}^{\infty} x^{r} g(x) G(x)^{\alpha-1} \sin \left(\frac{\pi}{2} G(x)^{\alpha}\right)\left[\cos \left(\frac{\pi}{2} G(x)^{\alpha}\right)\right]^{i} d x
$$

Insert

$\sin \left(\frac{\pi}{2} G(x)^{\alpha}\right)=\frac{\sum_{j=0}^{\infty}(-1)^{j} 2^{-(2 j+1)}(\pi)^{(2 j+1)} G(x)^{\alpha(2 j+1)}}{(2 j+1) !}$.
And

$$
\left[\cos \left(\frac{\pi}{2} G(x)^{\alpha}\right)\right]^{i}=\sum_{k=0}^{\infty} b_{k}(i)\left(\frac{\pi}{2} G(x)^{\alpha}\right)^{2 k}
$$

In (21), we get

$$
\begin{aligned}
& \rho_{r, q}=\sum_{i, j, k=0}^{\infty} \alpha \beta c_{k}\left(\begin{array}{c}
\beta(s+1)-1 \\
i
\end{array}\right) \frac{(-1)^{(i+j)}(2)^{-(2 j+1)-1}(\pi)^{(2 j+2)}}{(2 j+1) !} \int_{-\infty}^{\infty} x^{r} g(x) G(x)^{2 \alpha(j+k+1)-1} d x, \\
& \rho_{r, q}=\sum_{i, j, k=0}^{\infty} U_{(i, j, k)} \int_{-\infty}^{\infty} x^{r}(2 \alpha(j+k+1)) g(x) G(x)^{2 \alpha(j+k+1)-1} d x,
\end{aligned}
$$

where

$$
U_{(i, j, k)}=\alpha \beta c_{k}\left(\begin{array}{c}
\beta(s+1)-1 \\
i
\end{array}\right) \frac{(-1)^{(i+j)}(2)^{-(2 j+2)}(\pi)^{(2 j+2)}}{(2 j+1) !(2 \alpha(j+k+1))} .
$$

In case of generalized distributions, the PWMs are helpful to find the quantiles and estimators of concerned parameters. Moreover, low variance values with no severe biases are additional properties of PWMs. The obtained estimators through PWMs can be efficiently and favorably set side by side with relevant MLEs. Research may be conducted in the future on both methods for ext cos- $\mathrm{G}$ family and derived models.

2.10. Stochastic Ordering. Now we derive a useful result on the stochastic ordering using an ext cos- $G$ family with common parameters $\alpha$ and $\beta$. See [40] for further details on stochastic ordering. In the following theorem, an ext cos- $\mathrm{G}$ family of distributions is ordered with respect to the 
strongest "likelihood ratio" ordering, which expresses the flexibility of two parameters for the ext cos- $G$ family of distributions $(\alpha, \beta)$.
Theorem. Let $X$ follow an ext cos- $G\left(\alpha_{1}, \beta_{1}\right)$ and $Y$ follow an ext cos- $G\left(\alpha_{2}, \beta_{2}\right)$. If $\alpha_{1}=\alpha_{2}=\alpha$ and $\beta_{1} \geq \beta_{2}$, then $X \leqslant_{l r}(Y)$.

Proof. We have the following likelihood ratio:

$$
\frac{f_{X}(x)}{f_{Y}(x)}=\frac{1 / 2 \pi \alpha_{1} \beta_{1} G(x)^{\alpha_{1}-1} g(x) \sin \left(1 / 2 \pi G(x)^{\alpha_{1}}\right)\left(1-\cos \left(1 / 2 \pi G(x)^{\alpha_{1}}\right)\right)^{\beta_{1}-1}}{1 / 2 \pi \alpha_{2} \beta_{2} G(x)^{\alpha_{2}-1} g(x) \sin \left(1 / 2 \pi G(x)^{\alpha_{2}}\right)\left(1-\cos \left(1 / 2 \pi G(x)^{\alpha_{2}}\right)\right)^{\beta_{2}-1}} .
$$

Putting $\alpha_{1}=\alpha_{2}=\alpha$, we get

$$
\begin{aligned}
& \frac{f_{X}(x)}{f_{Y}(x)}=\frac{\beta_{1}}{\beta_{2}}\left(1-\cos \left(\frac{1}{2} \pi G(x)^{\alpha}\right)\right)^{\beta_{1}-\beta_{2}}, \\
& \frac{\mathrm{d}}{\mathrm{d} x} \frac{f_{X}(x)}{f_{Y}(x)}=\frac{\beta_{1}}{\beta_{2}}\left(\beta_{1}-\beta_{2}\right)\left(1-\cos \left(\frac{1}{2} \pi G(x)^{\alpha}\right)\right)^{\left(\beta_{1}-\beta_{2}\right)^{-1} \frac{1}{2} \pi \alpha G(x)^{\alpha-1} g(x) \sin \left(\frac{1}{2} \pi G(x)^{\alpha}\right) .} \\
& \text { hen } \mathrm{d} / \mathrm{d} x f_{X}(x) / f_{Y}(x)<0, \text { which completes } \\
& \text { implies that } X \leqslant_{l r}(Y) \text { and similarly } \\
& \text { (Y), } X \leqslant_{\text {mrl }}(Y), X \leqslant_{s t}(Y) \text {. This property is a } \\
& \text { observe the constitutional characteristics of }
\end{aligned}
$$
the proof and implies that $X \leqslant{ }_{l r}(Y)$ and similarly $X \leqslant_{l r}(Y), X \leqslant_{h r}(Y), X \leqslant_{m r l}(Y), X \leqslant_{s t}(Y)$. This property is a widget used to observe the constitutional characteristics of complex stochastic structure.

2.11. Order Statistics. In a number of fields of statistical methods and theory, especially in applications of survival testing studies, order statistics naturally arise. For more details, please consult the book of [41]. Here, we presented the order statistics of an ext cos- $G$ family of distributions. Suppose $X_{1}, \ldots, X_{n}$ be a random sample from an ext cos-G family; then $i$-th order statistic density is 
After applying the power series expansion, we get

$$
\left(1-\cos \left(\frac{\pi}{2} G(x)^{\alpha}\right)\right)^{\delta(\beta-1)}=\sum_{j=0}^{\infty}(-1)^{j} \sum_{k=0}^{\infty}\left(\begin{array}{c}
\delta(\beta-1) \\
j
\end{array}\right) C_{k} G(x)^{2 k \alpha}
$$

Finally, the Rényi entropy becomes

$$
I_{\delta}(f)=\frac{1}{1-\delta} \log \left[\sum_{j, k=0}^{\infty} D_{j, k, \delta} \int_{-\infty}^{\infty} g(x)^{\delta} G(x)^{\delta(2 k+\alpha-1)}\left(\sin \left(\frac{\pi}{2} G(x)^{\alpha}\right)\right)^{\delta}\right]
$$

where $D_{j, k, \delta}=(\pi / 2)^{\delta}(\alpha)^{\delta}(\beta)^{\delta}(-1)^{j}\left(\begin{array}{c}\delta(\beta-1) \\ j\end{array}\right) C_{k}$.

\section{An Extended Cosine Weibull \\ (ECW) Distribution}

In this section, the ECW model with its properties is presented.
3.1. Main Functions of ECW with Graphs. Now, using Weibull distribution (as baseline) having the cdf $G(x)=1-\mathrm{e}^{-\lambda x^{\sigma}}, \lambda, \sigma, x>0$, we presented a new model having right skew and heavy tail characteristics and extended flexibility compared to the former Weibull model. Hereafter, this will be called an Extended Cosine Weibull (ECW) $(\alpha, \beta, \lambda, \sigma)$ distribution. By using (2), the main functions of the newly presented distribution are

$$
\begin{aligned}
& F(x)=\left[1-\cos \left(\frac{\pi}{2}\left(1-\mathrm{e}^{-\lambda x^{\sigma}}\right)^{\alpha}\right)\right]^{\beta}, \quad x>0, \\
& f(x)=\frac{\pi}{2} \alpha \beta \lambda \sigma x^{\sigma-1} \mathrm{e}^{-\lambda x^{\sigma}}\left(1-\mathrm{e}^{-\lambda x^{\sigma}}\right)^{\alpha-1} \sin \left(\frac{\pi}{2}\left(1-\mathrm{e}^{-\lambda x^{\sigma}}\right)^{\alpha}\right)\left[1-\cos \left(\frac{\pi}{2}\left(1-\mathrm{e}^{-\lambda x^{\sigma}}\right)^{\alpha}\right)\right]^{\beta-1}, \\
& h(x)=\frac{\pi / 2 \alpha \beta \lambda \sigma x^{\sigma-1} \mathrm{e}^{-\lambda x^{\sigma}}\left(1-\mathrm{e}^{-\lambda x^{\sigma}}\right)^{\alpha-1} \sin \left(\pi / 2\left(1-\mathrm{e}^{-\lambda x^{\sigma}}\right)^{\alpha}\right)\left[1-\cos \left(\pi / 2\left(1-\mathrm{e}^{-\lambda x^{\sigma}}\right)^{\alpha}\right)\right]^{\beta-1}}{1-\left[1-\cos \left(\pi / 2\left(1-\mathrm{e}^{-\lambda x^{\sigma}}\right)^{\alpha}\right)\right]^{\beta}} .
\end{aligned}
$$

Figure 1 displays the exhibiting density shapes of the proposed model.

Figure 2 displays the exhibiting hazard rate function shapes of the proposed model.
3.2. Useful Reliability Functions of ECW. The important reliability functions are

$$
\begin{aligned}
& S(x)=1-\left[1-\cos \left(\frac{\pi}{2}\left(1-e^{-\lambda x^{\sigma}}\right)^{\alpha}\right)\right]^{\beta}, \\
& r(x)=\frac{\pi / 2 \alpha \beta \lambda \sigma x^{\sigma-1} \mathrm{e}^{-\lambda x^{\sigma}}\left(1-e^{-\lambda x^{\sigma}}\right)^{\alpha-1} \sin \left(\pi / 2\left(1-e^{-\lambda x^{\sigma}}\right)^{\alpha}\right)\left[1-\cos \left(\pi / 2\left(1-e^{-\lambda x^{\sigma}}\right)^{\alpha}\right)\right]^{\beta-1}}{\left[1-\cos \left(\pi / 2\left(1-e^{-\lambda x^{\sigma}}\right)^{\alpha}\right)\right]^{\beta}}, \\
& H(x)=-\ln (S(x))=-\ln \left(1-\left[1-\cos \left(\frac{\pi}{2}\left(1-e^{-\lambda x^{\sigma}}\right)^{\alpha}\right)\right]^{\beta}\right),
\end{aligned}
$$



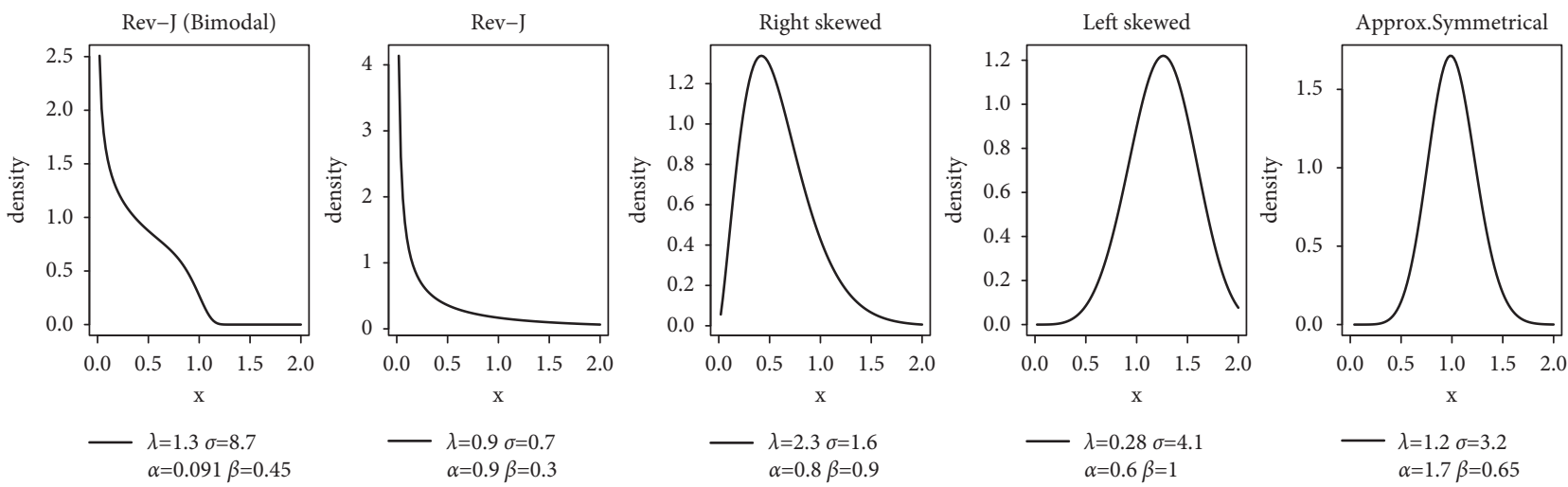

Figure 1: Some labeled density curves of the ECW.
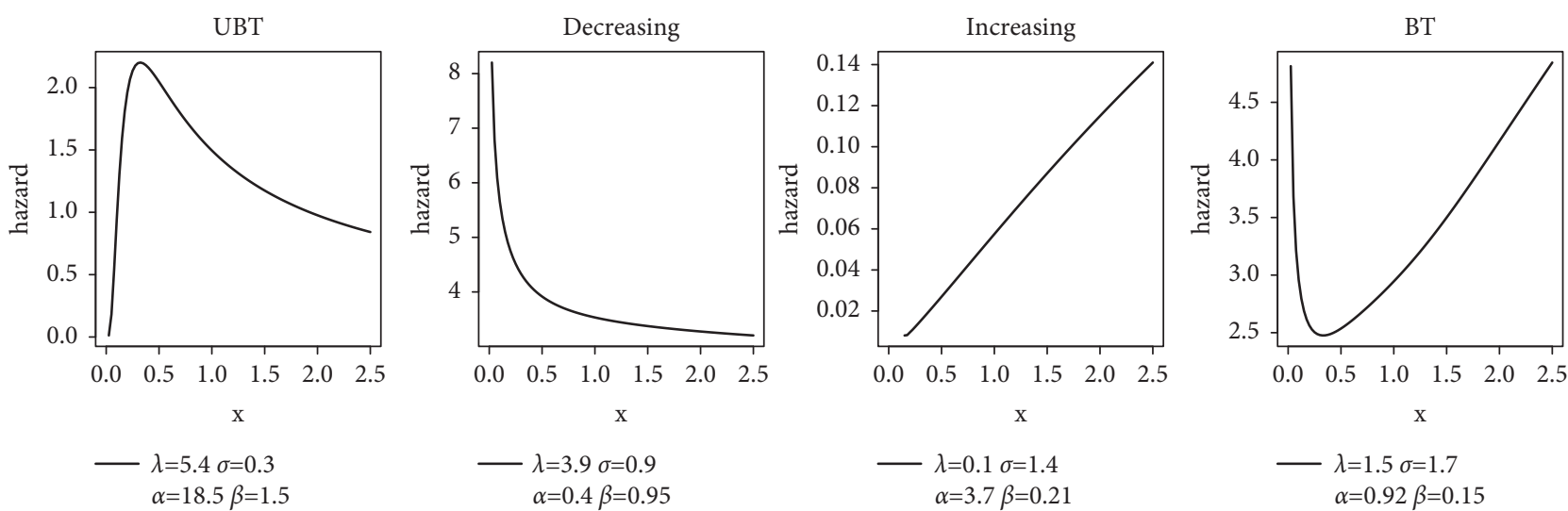

FIGURE 2: Some labeled hazard function curves of the ECW.

$$
\begin{aligned}
m(x) & =\frac{1-\left[1-\cos \left(\pi / 2\left(1-e^{-\lambda x^{\sigma}}\right)^{\alpha}\right)\right]^{\beta}}{\pi / 2 \alpha \beta \lambda \sigma x^{\sigma-1} \mathrm{e}^{-\lambda x^{\sigma}}\left(1-e^{-\lambda x^{\sigma}}\right)^{\alpha-1} \sin \left(\pi / 2\left(1-e^{-\lambda x^{\sigma}}\right)^{\alpha}\right)\left[1-\cos \left(\pi / 2\left(1-e^{-\lambda x^{\sigma}}\right)^{\alpha}\right)\right]^{\beta-1}}, \\
\bar{G}(x, \alpha, \beta, \lambda, \sigma \mid t) & =\frac{\bar{G}((x+t), \alpha, \beta, \lambda, \sigma)}{\bar{G}(t, \alpha, \beta, \lambda, \sigma)}=\frac{1-\left(1-\cos \left(\pi / 2\left(1-e^{-\lambda(x+t)^{\sigma}}\right)^{\alpha}\right)\right)^{\beta}}{1-\left(1-\cos \left(\pi / 2\left(1-e^{-\lambda t^{\sigma}}\right)^{\alpha}\right)\right)^{\beta}}, \\
e(x) & =\frac{\partial}{\partial(\ln (x))}\left[1-\cos \left(\pi / 2\left(1-e^{-\lambda x^{\sigma}}\right)^{\alpha}\right)\right]^{\beta} .
\end{aligned}
$$

3.3. Residual and Reverse Residual Life. In reliability theory and risk management, the residual life offers wide applications. Let $X$ denote the lifetime of a unit and $X \geq 0$ with $P(X=1)$; then similar to residual lifetime random variable, $X_{t}=(t-X \mid X \leq t)$, for a fixed $t>0$, named as "time since failure."

Let $R_{t}(x)$ denote the residual lifetime of the ECW random variable $X$, which is

$$
R_{t}(x)=\frac{1-\left(1-\cos \left(\pi / 2\left(1-e^{-\lambda(x+t)^{\sigma}}\right)^{\alpha}\right)\right)^{\beta}}{1-\left(1-\cos \left(\pi / 2\left(1-e^{-\lambda x^{\sigma}}\right)^{\alpha}\right)\right)^{\beta}} .
$$

In addition, the reversed hazard rate function $\bar{R}_{t}(x)$ has attracted the attention of several practitioners in the recent past, which is derived as

$$
\bar{R}_{t}(x)=\frac{\left.1-\left(1-\cos \left(\pi / 2\left(1-e^{-\lambda(x-t)^{\sigma}}\right)\right)^{\alpha}\right)\right)^{\beta}}{1-\left(1-\cos \left(\pi / 2\left(1-e^{-\lambda x^{\sigma}}\right)^{\alpha}\right)\right)^{\beta}} .
$$

3.4. Quantile Function, Quantile Density Function, Median, Skewness, and Kurtosis. By virtue of (36), the qf of ECW distribution is 


$$
Q_{F}(u)=\left[\frac{-1}{\lambda} \log \left(1-\left(\frac{2}{\pi} \cos ^{-1}\left(1-u^{1 / \beta}\right)\right)^{1 / \alpha}\right)\right]^{1 / \sigma} .
$$

$U$ is a uniform random variable. Moreover, the quantile density function (qdf) of ECW distribution is

$$
Q^{\prime}(u)=\frac{2 u^{(1-\beta / \beta)}\left(\pi / 2 \cos ^{-1}\left(1-u^{1 / \beta}\right)\right)^{(1-\alpha / \alpha)}\left(-1 / \lambda \log \left(1-\left(\pi / 2 \cos ^{-1}\left(1-u^{1 / \beta}\right)\right)^{1 / \alpha}\right)\right)^{1-\sigma / \sigma}}{\pi \lambda \beta \sigma \alpha \sqrt{\left(1-\left(1-u^{1 / \beta}\right)^{2}\right)}\left(1-\left(\pi / 2 \cos ^{-1}\left(1-u^{1 / \beta}\right)\right)^{1 / \alpha}\right)} .
$$

The median of ECW distribution is

$$
\text { Median }=Q_{F}(0.5)=\left(\frac{-1}{\lambda} \log \left(1-\left(2 / \pi \cos ^{-1}\left(1-(0.5)^{1 / \beta}\right)\right)^{1 / \alpha}\right)\right)^{1 / \sigma}
$$

Table 5 describes the numerical values of the first quartile $Q_{1}$, second quartile $Q_{2}$, third quartile $Q_{3}$, skewness (Bowley), and kurtosis (Moors) of ECW distribution for selected values for $\alpha, \beta, \lambda$, and $\sigma$.

The numerical results in Tables 5 and 6 are interpreted as follows:

(i) When $\beta, \lambda, \sigma$ are put constant and $\alpha$ is increased, then skewness and kurtosis $\longrightarrow 0$.

(ii) When $\alpha, \lambda, \sigma$ are put constant and $\beta$ is increased, skewness and kurtosis $\longrightarrow 0$.

(iii) When $\alpha, \beta, \sigma$ are put constant and $\lambda$ is increased, no significant effect is observed on skewness and kurtosis. (iv) When $\alpha, \beta, \lambda$ are put constant and $\sigma$ is increased, skewness and kurtosis $\longrightarrow 0$.

3.5. MacGillivary's Skewness. MacGillivary (1986) developed a graphical method to access the skewness of the model using quantile function, which is defined as follows:

$$
\delta(p)=\frac{\delta_{(1)}(\alpha, \beta, \sigma, \lambda)}{\delta_{(2)}(\alpha, \beta, \sigma, \lambda)}=\frac{Q_{(1-p)}+Q_{(p)}-2 Q_{(1 / 2)}}{Q_{(1-p)}-Q_{(p)}},
$$

where $p \varepsilon(0,1)$ and $Q($.$) are the qf stated in (42)$.

$$
\begin{aligned}
\delta_{(1)}(\alpha, \beta, \sigma, \lambda)= & {\left[\frac{-1}{\lambda} \log \left(1-\left(\frac{2}{\pi} \cos ^{-1}\left(p^{1 / \beta}\right)\right)^{1 / \alpha}\right)\right]^{1 / \sigma}+\left[\frac{-1}{\lambda} \log \left(1-\left(\frac{2}{\pi} \cos ^{-1}\left(1-p^{1 / \beta}\right)\right)^{1 / \alpha}\right)\right]^{1 / \sigma} } \\
& -2\left[\frac{-1}{\lambda} \log \left(1-\left(\frac{2}{\pi} \cos ^{-1}\left(0.5^{1 / \beta}\right)\right)^{1 / \alpha}\right)\right]^{1 / \sigma} \cdot \\
\delta_{(2)}(\alpha, \beta, \sigma, \lambda)= & {\left[\frac{-1}{\lambda} \log \left(1-\left(\frac{2}{\pi} \cos ^{-1}\left(p^{1 / \beta}\right)\right)^{1 / \alpha}\right)\right]^{1 / \sigma}+\left[\frac{-1}{\lambda} \log \left(1-\left(\frac{2}{\pi} \cos ^{-1}\left(1-p^{1 / \beta}\right)\right)^{1 / \alpha}\right)\right]^{1 / \sigma} . }
\end{aligned}
$$

MacGillivary measure of skewness $\delta(p)$ is purely based on qf and hence can efficiently describe the parametric effect on the skewness. Figure 3 (left) shows that if $\alpha, \lambda, \sigma$ are put constant and $\beta$ is increased, then skewness $\longrightarrow 0$, while Figure 3 (right) shows that if $\alpha, \lambda, \beta$ are put constant and $\sigma$ is increased, then skewness $\longrightarrow 0$.

3.6. Skewness and Kurtosis via 3D Graphs. Recently, the trend in graphical representation has been changed, and now visual representation gets popularity and is preferred on numerical and tabular representation. The 3D graphs presented in the following clearly attest to the change in kurtosis and skewness after altering the parametric values for $\alpha$ and $\beta$.

In Figure 4 , it is clear that as $\alpha$ and $\beta$ are increased, the skewness is decreased (symmetry is increased), and in the same pattern, the kurtosis is reduced (normality is increased).

\section{Estimation and Inference of ECW}

Let $x_{1}, \ldots, x_{n}$ be a random sample of size $n$, which follow the ECW model. Then the log-likelihood function based on (37) is 
TABle 5: First quartile, median, third quartile, Bowley skewness, and Moors kurtosis of ECW $(\alpha, \beta, \lambda, \sigma)$ distribution for the following selected parameters' values in order $(\alpha, \beta, \lambda, \sigma)$ : (i) $(2.5,0.5,1.5,0.7)$, (ii) $(2.5,1.0,0.5,1.2)$, (iii) $(2.5,2.0,1.5,1.5)$, (iv) $(3.0,2.5,2.0,2.0)$, and (v) $(6.0,1.0,5.0,3.0)$.

\begin{tabular}{lcccrr}
\hline & $Q(1 / 4)$ & Med & $Q(3 / 4)$ & $B$ & \\
\hline (i) & 0.409 & 0.834 & 1.576 & 0.272 & 0.128 \\
(ii) & 2.247 & 3.041 & 4.067 & 0.097 & 1.394 \\
(iii) & 1.170 & 1.413 & 1.708 & 0.076 & 1.273 \\
(iv) & 1.065 & 1.205 & 1.368 & 0.053 & 1.249 \\
(v) & 0.750 & 0.817 & 0.892 & 1.249 \\
\hline
\end{tabular}

TABLE 6: Some moments of ECW $(\alpha, \beta, \lambda, \sigma)$ distribution for the following selected parameters' values in order $(\alpha, \beta, \lambda, \sigma)$ : (i) $(2.5,0.5,1.5,0.7)$, (ii) $(2.5,1.0,0.5,1.2)$, (iii) $(2.5,2.0,1.5,1.5)$, (iv) $(3.0,2.5,2.0,2.0)$, and (v) $(6.0,1.0,5.0,3.0)$.

\begin{tabular}{lccccccccc}
\hline & $\mathbb{E}(X)$ & $\mathbb{E}\left(X^{2}\right)$ & $\mathbb{E}\left(X^{3}\right)$ & $\mathbb{E}\left(X^{4}\right)$ & $\mathbb{V}(X)$ & $\sigma(X)$ & $\mathrm{CV}$ & $\mathrm{CS}$ & $\mathrm{CK}$ \\
\hline (i) & 0.505 & 1.011 & 4.867 & 43.595 & 0.756 & 0.870 & 1.723 & 5.462 & 61.384 \\
(ii) & 0.400 & 0.313 & 0.369 & 0.585 & 0.152 & 0.390 & 0.976 & 2.048 & 9.366 \\
(iii) & 0.868 & 0.812 & 0.815 & 0.873 & 0.059 & 0.242 & 0.279 & 0.571 \\
(iv) & 0.913 & 0.862 & 0.840 & 0.845 & 0.028 & 0.167 & 0.182 & 0.457 & 3.483 \\
(v) & 0.633 & 0.444 & 0.346 & 0.302 & 0.043 & 0.207 & 0.326 & 1.241 & 5.739 \\
\hline
\end{tabular}

$\ell(\alpha, \beta, \lambda, \sigma)=n \log \alpha+n \log \beta+n \log \lambda+n \log \sigma$

$$
\begin{aligned}
& +(\beta+1) n \log \left[1-\cos \left(\frac{1}{2} \pi\left(1-e^{-\lambda x^{\sigma}}\right)^{\alpha}\right)\right] \\
& +n \log \left[\sin \left(\frac{1}{2} \pi\left(1-e^{-\lambda x^{\sigma}}\right)^{\alpha}\right)\right]-n \lambda x^{\sigma}+n(\sigma-1) \log \left[e^{-\lambda x^{\sigma}}\left(e^{\lambda x^{\sigma}}-1\right)\right] \\
& -n(\sigma-1) \log x+n \log \left(\frac{\pi}{2}\right) .
\end{aligned}
$$

The maximum likelihood estimators (MLEs) are the solution of the nonlinear equations: $\partial \ell(\alpha, \beta, \lambda, \sigma) / \partial \alpha=0$, $\partial \ell(\alpha, \beta, \lambda, \sigma) / \partial \beta=0, \partial \ell(\alpha, \beta, \lambda, \sigma) / \partial \lambda=0$, and $\partial \ell(\alpha, \beta, \lambda, \sigma) /$ $\partial \sigma=0$ with

$$
\begin{aligned}
& \partial \ell \frac{(\alpha, \beta, \lambda, \sigma)}{\partial \alpha}=\frac{n}{\alpha} \\
& +\frac{1}{2} \pi n\left(1-\mathrm{e}^{-\lambda x^{\sigma}}\right)^{\alpha} \log \left(1-\mathrm{e}^{-\lambda x^{\sigma}}\right)\left((\beta+1) \cot \left(\frac{1}{4} \pi\left(1-\mathrm{e}^{-\lambda x^{\sigma}}\right)^{\alpha}\right)\right) \\
& +\left(\cot \left(\frac{1}{2} \pi\left(1-\mathrm{e}^{-\lambda x^{\sigma}}\right)^{\alpha}\right)\right) \\
& \partial \ell \frac{(\alpha, \beta, \lambda, \sigma)}{\partial \beta}=n\left(\frac{1}{\beta}+\log \left(\sin ^{2}\left(\frac{1}{4} \pi\left(1-\mathrm{e}^{-\lambda x^{\sigma}}\right)^{\alpha}\right)\right)+\log (2)\right) \\
& \partial \ell \frac{(\alpha, \beta, \lambda, \sigma)}{\partial \lambda}=\frac{n\left(\pi \alpha \lambda x^{\sigma}\left(1-\mathrm{e}^{-\lambda x^{\sigma}}\right)^{\alpha} \tan \left(1 / 4 \pi\left(1-\mathrm{e}^{-\lambda x^{\sigma}}\right)^{\alpha}\right)\left((2 \beta+3) \cot ^{2}\left(1 / 4 \pi\left(1-\mathrm{e}^{-\lambda x^{\sigma}}\right)^{\alpha}\right)-1\right)\right)}{4 \lambda\left(\mathrm{e}^{\lambda x^{\sigma}}-1\right)} \\
& +\frac{4\left(\lambda \sigma x^{\sigma}+\mathrm{e}^{\lambda x^{\sigma}}\left(1-\lambda x^{\sigma}\right)-1\right)}{4 \lambda\left(\mathrm{e}^{\lambda x^{\sigma}}-1\right)} \\
& \partial \ell \frac{(\alpha, \beta, \lambda, \sigma)}{\partial \sigma}=n\left(\frac{1}{\sigma}+\log \left(1-\mathrm{e}^{-\lambda x^{\sigma}}\right)-\log (x)\right)+\frac{\lambda n x^{\sigma} \log (x)\left(4 \sigma+\pi \alpha\left(1-\mathrm{e}^{-\lambda x^{\sigma}}\right)^{\alpha} \tan \left(1 / 4 \pi\left(1-\mathrm{e}^{-\lambda x^{\sigma}}\right)^{\alpha}\right)\right)}{4\left(\mathrm{e}^{\lambda x^{\sigma}}-1\right)} \\
& * \frac{\left((2 \beta+3) \cot ^{2}\left(1 / 4 \pi\left(1-\mathrm{e}^{-\lambda x^{\sigma}}\right)^{\alpha}\right)-1\right)-4 \mathrm{e}^{\lambda x^{\sigma}}}{4\left(\mathrm{e}^{\lambda x^{\sigma}}-1\right)} \text {. }
\end{aligned}
$$



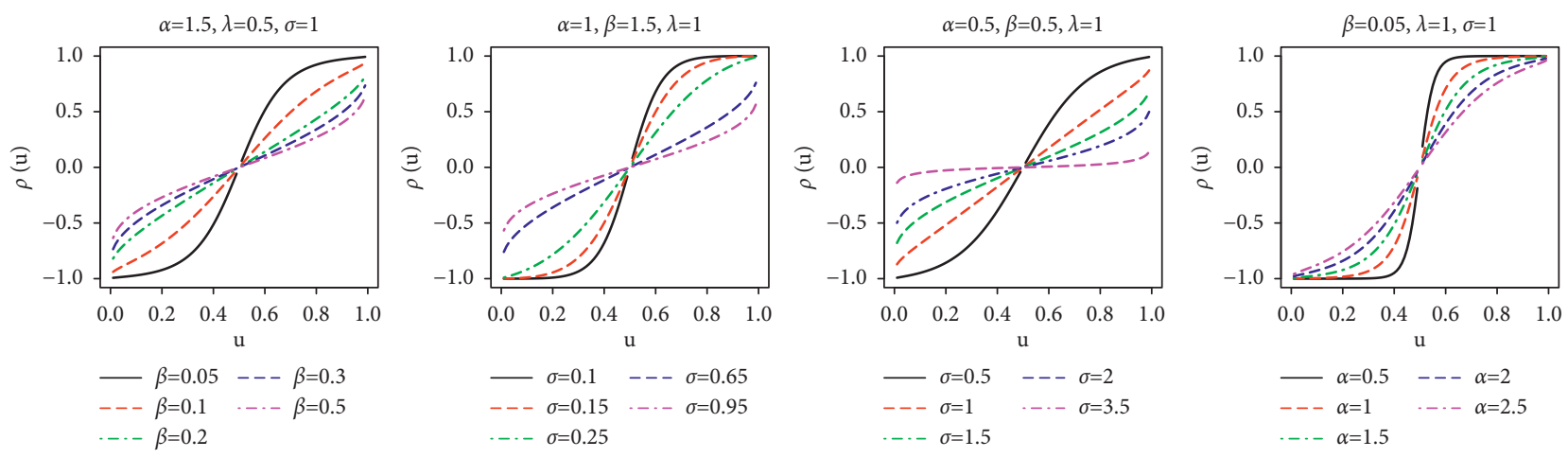

Figure 3: MacGillivary skewness graphs for specific parametric values.
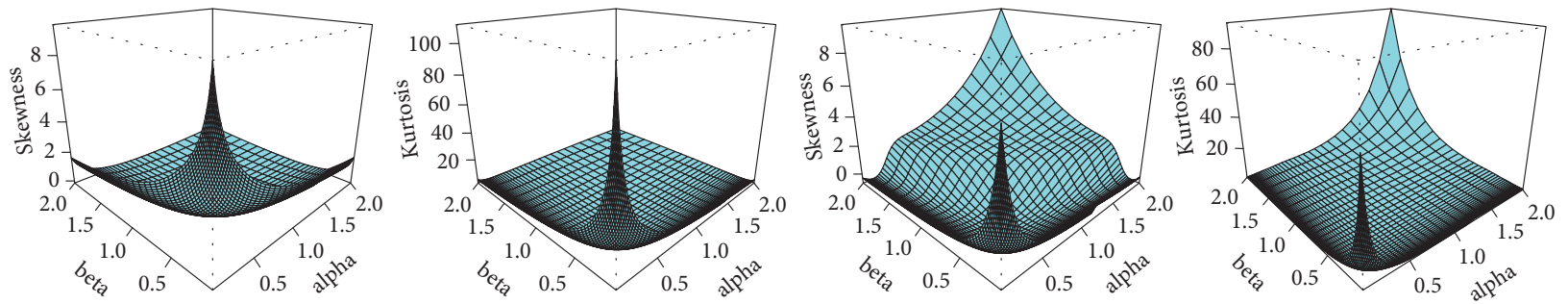

Figure 4: 3D graphs for the skewness (left) and the kurtosis (right) using different parametric values.

The MLEs $(\hat{\alpha}, \widehat{\beta}, \hat{\lambda}, \widehat{\sigma})$ can be obtained by setting the above equations to zero and solving them simultaneously.

4.1. Simulation Study. To check the convergence of the MLEs, a Monte Carlo simulation study is carried out at $N=$ 1,000 repetition times with $n=50,100,200,300,500,1000$ sample sizes and the following parametric combinations: I: $\alpha=2.0, \beta=1.5, \lambda=0.5$, and $\sigma=1.2$, II: $\alpha=2.5, \beta=3.5$, $\lambda=1.5$, and $\sigma=2.2$, III: $\alpha=1.5, \beta=4.0, \quad \lambda=2.5$, and $\sigma=2.0$, and IV: $\alpha=3.5, \beta=4.0, \lambda=2.0$, and $\sigma=3.0$. The empirical biases, mean squared errors (MSEs), coverage probabilities (CP) of the $95 \%$ two-sided confidence intervals for some sample sizes, the lower bound (L.bound) of these intervals, and their upper bounds (U.bound) for the model ECW parameters can be found in Tables 7 and 8 .

Specifically, concerning the theory, we see that the biases and MSEs decline with expanding sample size. Additionally, the CPs of the confidence intervals are very near to the $95 \%$ nominal levels. Accordingly, the MLEs and their asymptotic outcomes can be utilized for estimating and constructing confidence intervals for the model parameters.

In Figure 5, the graphs (left and middle) clearly depict that biases and MSE(s) are approaching zero, while the graphs (right) show that CP is overall increasing.

4.2. Analysis of ECW Model. Considering ECW $(\alpha, \beta, \lambda, \sigma)$ distribution as a statistical model, the MLEs are calculated, and significant goodness-of-fit criterion results, like the loglikelihood function checked at the MLEs $(\widehat{\ell})$, Cramér-von Mises $\left(W^{*}\right)$, Anderson-Darling $\left(A^{*}\right), p$-values, and Kolmogorov-Smirnov (K-S), are computed for comparison with configured models. Reference [42] provided good details about $A^{*}$ and $W^{*}$ statistics. Here, we compare the ECW distribution with six models, namely, Exponentiated Kumaraswamy Weibull (EKumW), Beta Weibull (BW), Topp-Leone Weibull (TLW), Logistic Weibull (LW), Cosine Weibull (CW), and Weibull (W).

4.2.1. First Application: Cancer Patients' Survival Times Data. The first data represent 121 breast cancer patients' survival times during a specific period from 1929 to 1938 . The data source is [43] and Ramos et al. (2013) and Tahir et al. (2014) studied these datasets. The observations are listed as follows:

$0.3,0.3,4.0,5.0,5.6,6.2,6.3,6.6,6.8,7.4,7.5,8.4,8.4$, $10.3,11.0,11.8,12.2,12.3,13.5,14.4,14.4,14.8,15.5,15.7$, $16.2,16.3,16.5,16.8,17.2,17.3,17.5,17.9,19.8,20.4,20.9$, $21.0,21.0,21.1,23.0,23.4,23.6,24.0,24.0,27.9,28.2,29.1$, $30.0,31.0,31.0,32.0,35.0,35.0,37.0,37.0,37.0,38.0,38.0$, 38.0, 39.0, 39.0, 40.0, 40.0, 40.0, 41.0, 41.0, 41.0, 42.0, 43.0, $43.0,43.0,44.0,45.0,45.0,46.0,46.0,47.0,48.0,49.0,51.0$, $51.0,51.0,52.0,54.0,55.0,56.0,57.0,58.0,59.0,60.0,60.0$, $60.0,61.0,62.0,65.0,65.0,67.0,67.0,68.0,69.0,78.0,80.0$, 83.0, 88.0, 89.0, 90.0, 93.0, 96.0, 103.0, 105.0, 109.0, 109.0, $111.0,115.0,117.0,125.0,126.0,127.0,129.0,129.0,139.0$, and 154.0 .

The summary statistics are $\min . x=0.30, \max \cdot x=154$, $\mathrm{Q} 1=17.50$, median $=40.00$, mean $=46.33, Q 3=60.00$, $\mathrm{SD}=35.28, \quad$ range $=153.7, \quad$ skewness $=1.03, \quad$ and kurtosis $=0.35$.

Figure 6(a) presents the histogram of the first data, showing a heavy right tail, while the TTT plot is shown in Figure 6(b), which is concave only. The boxplot is displayed in Figure 6(c), while the kernel density is shown in 
TABLE 7: The bias, MSE, CP, L.bound, and U.bound obtained from simulation of ECW model.

\begin{tabular}{|c|c|c|c|c|c|c|c|c|c|c|c|c|c|}
\hline$n$ & & Initial & Bias & MSE & $\mathrm{CP}$ & L.bound & U.bound & Initial & Bias & MSE & $\mathrm{CP}$ & L.bound & U.bound \\
\hline \multirow[t]{4}{*}{50} & $\alpha$ & 2.0 & 5.514 & 621.443 & 0.93 & 88.695 & 103.723 & 2.5 & 6.748 & 393.707 & 0.94 & 138.835 & 157.332 \\
\hline & $\beta$ & 1.5 & 2.621 & 60.221 & 0.98 & 50.665 & 58.908 & 3.5 & 3.295 & 456.436 & 0.91 & 178.791 & 192.380 \\
\hline & $\lambda$ & 0.5 & 0.373 & 0.999 & 0.88 & 1.304 & 3.050 & 1.5 & 0.181 & 1.846 & 0.88 & 2.012 & 5.375 \\
\hline & $\sigma$ & 1.2 & 0.098 & 0.454 & 0.98 & 0.180 & 2.618 & 2.2 & 0.474 & 2.170 & 1.00 & 0.436 & 5.605 \\
\hline \multirow[t]{4}{*}{100} & $\alpha$ & 2.0 & 1.961 & 77.440 & 0.94 & 20.849 & 28.770 & 2.5 & 5.372 & 250.029 & 0.96 & 61.020 & 76.764 \\
\hline & $\beta$ & 1.5 & 2.675 & 21.293 & 0.97 & 36.446 & 44.795 & 3.5 & 2.565 & 188.506 & 0.90 & 84.615 & 96.745 \\
\hline & $\lambda$ & 0.5 & 0.210 & 0.392 & 0.90 & 0.525 & 1.944 & 1.5 & 0.227 & 1.197 & 0.92 & 0.851 & 4.305 \\
\hline & $\sigma$ & 1.2 & 0.028 & 0.168 & 0.96 & 0.381 & 2.077 & 2.2 & 0.138 & 0.703 & 0.99 & 0.586 & 4.128 \\
\hline \multirow[t]{4}{*}{200} & $\alpha$ & 2.0 & 1.164 & 23.513 & 0.92 & 11.341 & 17.669 & 2.5 & 3.371 & 89.962 & 0.90 & 25.701 & 37.443 \\
\hline & $\beta$ & 1.5 & 2.418 & 20.113 & 0.94 & 26.634 & 34.471 & 3.5 & 2.124 & 30.753 & 0.88 & 49.610 & 60.858 \\
\hline & $\lambda$ & 0.5 & 0.127 & 0.143 & 0.95 & 0.156 & 1.408 & 1.5 & 0.177 & 0.557 & 0.96 & 0.181 & 3.413 \\
\hline & $\sigma$ & 1.2 & -0.012 & 0.072 & 0.97 & 0.614 & 1.763 & 2.2 & 0.019 & 0.306 & 0.98 & 1.013 & 3.425 \\
\hline \multirow[t]{4}{*}{300} & $\alpha$ & 2.0 & 0.919 & 14.153 & 0.93 & 9.100 & 14.938 & 2.5 & 3.660 & 87.361 & 0.95 & 21.762 & 34.082 \\
\hline & $\beta$ & 1.5 & 2.130 & 15.485 & 0.94 & 20.101 & 27.361 & 3.5 & 1.579 & 22.705 & 0.84 & 35.641 & 45.799 \\
\hline & $\lambda$ & 0.5 & 0.101 & 0.088 & 0.96 & 0.069 & 1.222 & 1.5 & 0.139 & 0.377 & 0.97 & 0.298 & 3.018 \\
\hline & $\sigma$ & 1.2 & -0.022 & 0.046 & 0.96 & 0.710 & 1.647 & 2.2 & -0.005 & 0.186 & 0.98 & 1.219 & 3.171 \\
\hline \multirow[t]{4}{*}{500} & $\alpha$ & 2.0 & 0.579 & 8.975 & 0.90 & 6.475 & 11.632 & 2.5 & 2.416 & 42.403 & 0.92 & 14.747 & 24.579 \\
\hline & $\beta$ & 1.5 & 2.196 & 16.461 & 0.93 & 17.327 & 24.719 & 3.5 & 1.578 & 23.198 & 0.87 & 29.977 & 40.134 \\
\hline & $\lambda$ & 0.5 & 0.067 & 0.044 & 0.97 & 0.108 & 1.035 & 1.5 & 0.107 & 0.207 & 0.96 & 0.554 & 2.679 \\
\hline & $\sigma$ & 1.2 & -0.018 & 0.025 & 0.97 & 0.814 & 1.549 & 2.2 & -0.021 & 0.120 & 0.97 & 1.414 & 2.945 \\
\hline \multirow[t]{4}{*}{1000} & $\alpha$ & 2.0 & 0.558 & 6.650 & 0.94 & 5.001 & 10.116 & 2.5 & 2.156 & 34.204 & 0.95 & 10.189 & 19.500 \\
\hline & $\beta$ & 1.5 & 1.701 & 12.264 & 0.93 & 11.152 & 17.554 & 3.5 & 1.391 & 19.268 & 0.85 & 21.256 & 31.039 \\
\hline & $\lambda$ & 0.5 & 0.050 & 0.022 & 0.98 & 0.218 & 0.884 & 1.5 & 0.077 & 0.099 & 0.98 & 0.813 & 2.341 \\
\hline & $\sigma$ & 1.2 & -0.022 & 0.013 & 0.97 & 0.912 & 1.445 & 2.2 & -0.031 & 0.055 & 0.97 & 1.620 & 2.718 \\
\hline
\end{tabular}

TABle 8: The bias, MSE, CP, L.bound, and U.bound obtained from simulation of ECW model.

\begin{tabular}{cccccccccccccc}
\hline 50 & $\alpha$ & 1.5 & 4.090 & 129.984 & 0.92 & 64.873 & 76.054 & 3.5 & 10.379 & 1159.982 & 0.93 & 277.221 & 304.979 \\
& $\beta$ & 4.0 & 1.917 & 99.612 & 0.90 & 124.588 & 136.423 & 4.0 & 4.041 & 803.360 & 0.90 & 233.217 & 249.300 \\
& $\lambda$ & 2.5 & 0.118 & 2.090 & 0.93 & 1.222 & 6.448 & 2.0 & 0.089 & 2.408 & 0.89 & 2.441 & 6.620 \\
& $\sigma$ & 2.0 & 0.422 & 1.915 & 1.00 & 0.360 & 4.997 & 3.0 & 0.712 & 3.824 & 1.00 & 0.672 & 7.870 \\
\hline \multirow{2}{*}{100} & $\alpha$ & 1.5 & 3.286 & 81.199 & 0.89 & 31.863 & 41.435 & 3.5 & 11.364 & 1155.276 & 0.90 & 197.695 & 227.423 \\
& $\beta$ & 4.0 & 1.648 & 60.978 & 0.88 & 70.671 & 81.968 & 4.0 & 3.941 & 659.501 & 0.88 & 170.913 & 186.795 \\
& $\lambda$ & 2.5 & 0.232 & 1.333 & 0.95 & 0.310 & 5.458 & 2.0 & 0.265 & 2.066 & 0.92 & 1.091 & 5.619 \\
& $\sigma$ & 2.0 & 0.107 & 0.560 & 0.98 & 0.565 & 3.661 & 3.0 & 0.236 & 1.482 & 0.99 & 0.740 & 5.832 \\
\hline 200 & $\alpha$ & 1.5 & 2.457 & 41.989 & 0.92 & 16.483 & 24.398 & 3.5 & 6.050 & 303.360 & 0.93 & 50.928 & 70.028 \\
& $\beta$ & 4.0 & 1.448 & 28.288 & 0.86 & 48.323 & 59.220 & 4.0 & 2.077 & 61.763 & 0.88 & 57.580 & 69.734 \\
& $\lambda$ & 2.5 & 0.159 & 0.688 & 0.98 & 0.830 & 4.510 & 2.0 & 0.169 & 0.874 & 0.96 & 0.220 & 4.343 \\
& $\sigma$ & 2.0 & 0.015 & 0.230 & 0.96 & 0.965 & 3.065 & 3.0 & 0.060 & 0.582 & 0.99 & 1.321 & 4.803 \\
\hline 300 & $\alpha$ & 1.5 & 2.150 & 28.857 & 0.93 & 12.673 & 19.972 & 3.5 & 5.660 & 191.644 & 0.94 & 34.477 & 52.798 \\
& $\beta$ & 4.0 & 1.267 & 27.004 & 0.85 & 38.657 & 49.191 & 4.0 & 1.363 & 26.229 & 0.86 & 41.528 & 52.254 \\
& $\lambda$ & 2.5 & 0.116 & 0.388 & 0.98 & 1.148 & 4.087 & 2.0 & 0.143 & 0.592 & 0.96 & 0.439 & 3.902 \\
& $\sigma$ & 2.0 & 0.000 & 0.141 & 0.97 & 1.148 & 2.853 & 3.0 & 0.034 & 0.432 & 0.97 & 1.608 & 4.460 \\
\hline 500 & $\alpha$ & 1.5 & 1.906 & 21.895 & 0.91 & 9.054 & 15.866 & 3.5 & 4.850 & 149.737 & 0.93 & 25.290 & 41.989 \\
& $\beta$ & 4.0 & 0.877 & 20.226 & 0.82 & 28.662 & 38.417 & 4.0 & 1.191 & 23.163 & 0.84 & 32.997 & 43.380 \\
& $\lambda$ & 2.5 & 0.079 & 0.229 & 0.97 & 1.437 & 3.722 & 2.0 & 0.079 & 0.350 & 0.97 & 0.629 & 3.536 \\
& $\sigma$ & 2.0 & -0.007 & 0.091 & 0.96 & 1.329 & 2.657 & 3.0 & -0.030 & 0.244 & 0.98 & 1.874 & 4.066 \\
\hline 1000 & $\alpha$ & 1.5 & 1.560 & 14.969 & 0.97 & 6.636 & 12.757 & 3.5 & 3.808 & 90.184 & 0.98 & 16.403 & 31.018 \\
& $\beta$ & 4.0 & 1.198 & 23.410 & 0.81 & 24.178 & 34.574 & 4.0 & 1.110 & 22.787 & 0.82 & 23.483 & 33.704 \\
& $\lambda$ & 2.5 & 0.066 & 0.115 & 0.98 & 1.717 & 3.414 & 2.0 & 0.068 & 0.146 & 0.98 & 1.113 & 3.027 \\
& $\sigma$ & 2.0 & -0.023 & 0.043 & 0.98 & 1.492 & 2.461 & 3.0 & -0.025 & 0.109 & 0.98 & 2.182 & 3.769 \\
\hline
\end{tabular}

Figure $6(\mathrm{~d})$, and the conclusion is that the first data is rightskewed. These graphics motivate the use of a model with a heavy right tail, such as the ECW model.

Table 9 lists the MLEs and their corresponding standard errors (in parentheses) of the model parameters for cancer patients' survival times data.
Table 10 lists the statistics $\widehat{\ell}, A^{*}, W^{*}, \mathrm{~K}-\mathrm{S}$, and $P$-value for cancer patients' survival times data.

Figure 7 (a) presents the plots of the estimated densities, Figure 7(b) shows the plots of the estimated distribution functions, and Figure 7(c) provides the P-P plots for cancer patients' survival times data. 

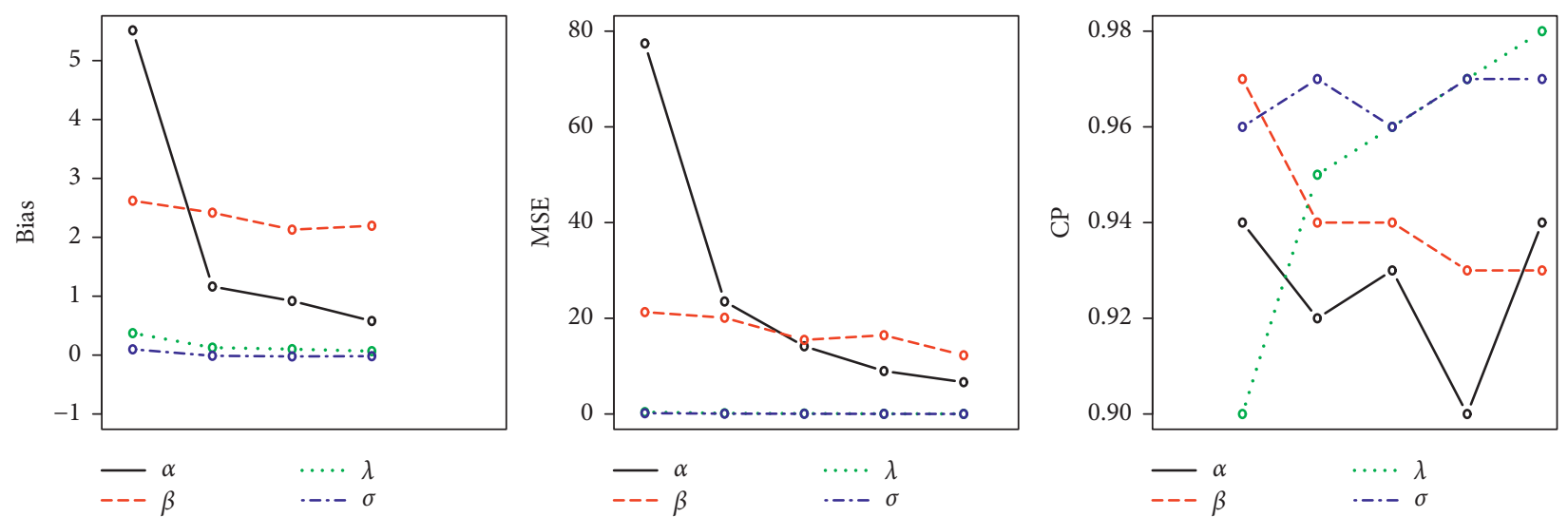

FIGURE 5: Graphs for bias (left), MSE (middle), and coverage probability (right).

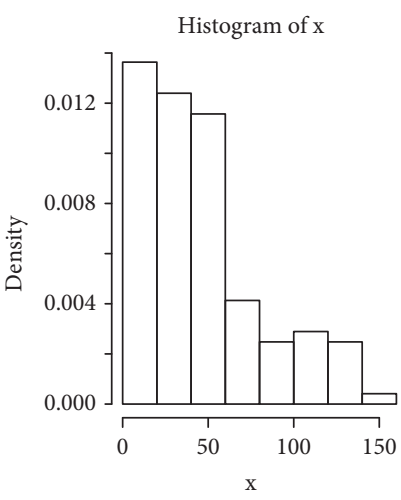

(a)

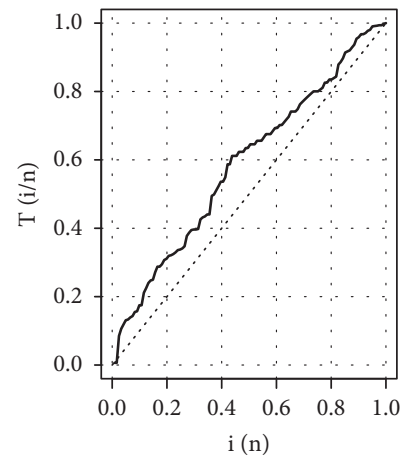

(b)

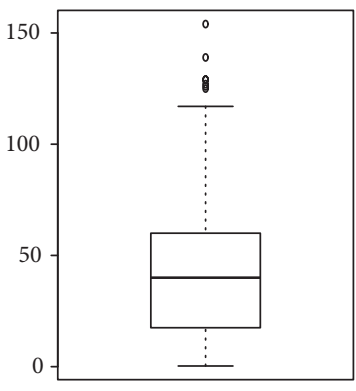

(c)

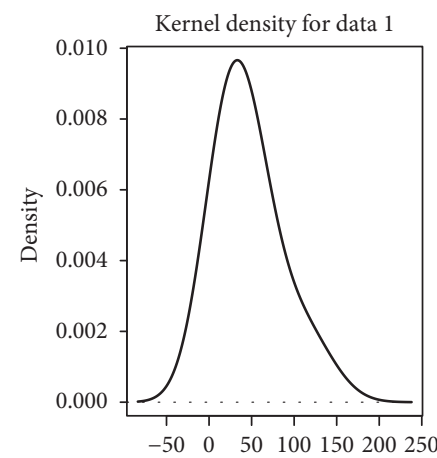

(d)

Figure 6: (a) Histogram. (b) TTT plot. (c) Boxplot. (d) Kernel density for cancer patients' survival times data.

TABLE 9: MLEs and their standard errors (in parentheses) for cancer patients' survival times data.

\begin{tabular}{|c|c|c|c|c|c|}
\hline Distribution & $\alpha$ & $\beta$ & $a$ & $b$ & $c$ \\
\hline \multirow[t]{2}{*}{ ECW } & 0.0069 & 1.2602 & 2.6688 & 0.116 & - \\
\hline & $(0.0007)$ & $(0.0048)$ & $(0.0048)$ & $(0.0225)$ & - \\
\hline \multirow[t]{2}{*}{ EKumW } & 0.0056 & 1.3753 & 1.0578 & 5.4583 & 0.8345 \\
\hline & (0.0019) & $(0.5426)$ & $(0.6979)$ & $(4.2773)$ & $(0.4278)$ \\
\hline \multirow[t]{2}{*}{ BW } & 0.9967 & 0.7769 & 0.0085 & 1.2817 & - \\
\hline & $(0.4467)$ & $(1.3907)$ & $(0.0047)$ & $(0.3401)$ & - \\
\hline \multirow[t]{2}{*}{ TLW } & 0.0061 & 1.1622 & 1.1852 & - & - \\
\hline & $(0.0021)$ & $(0.0728)$ & (0.173 9) & - & - \\
\hline \multirow[t]{2}{*}{ LW } & 0.6650 & 0.0936 & 2.7898 & - & - \\
\hline & $(0.9411)$ & $(0.3138)$ & (3.954 7) & - & - \\
\hline \multirow[t]{2}{*}{ CW } & 0.0426 & 0.9092 & - & - & - \\
\hline & $(0.0110)$ & $(0.0628)$ & - & - & - \\
\hline \multirow[t]{2}{*}{ W } & 0.0063 & 1.2953 & - & - & - \\
\hline & $(0.0018)$ & $(0.0684)$ & - & - & - \\
\hline
\end{tabular}

Table 10: The statistics $\widehat{\ell}, A^{*}, W^{*}, \mathrm{~K}-\mathrm{S}$, and $P$-value for cancer patients' survival times data.

\begin{tabular}{lcccrc}
\hline Distribution & $\hat{\ell}$ & $A^{*}$ & $W^{*}$ & K-S & $P$-value \\
\hline ECW & 579.1500 & 0.3945 & 0.0517 & 0.0588 & 0.7969 \\
EKumW & 579.9095 & 0.4297 & 0.0571 & 0.0591 & 0.0654 \\
BW & 579.1593 & 0.3983 & 0.0533 & 0.0702 & 0.6783 \\
TLW & 579.3489 & 0.3949 & 0.0536 & 0.0936 & 0.5899 \\
LW & 587.5996 & 1.2580 & 0.2097 & 0.0873 & 0.2387 \\
CW & 581.5372 & 0.5587 & 0.0860 & 0.0641 & 0.7139 \\
W & 579.0321 & 0.4000 & 0.0535 & & 0.7020 \\
\hline
\end{tabular}




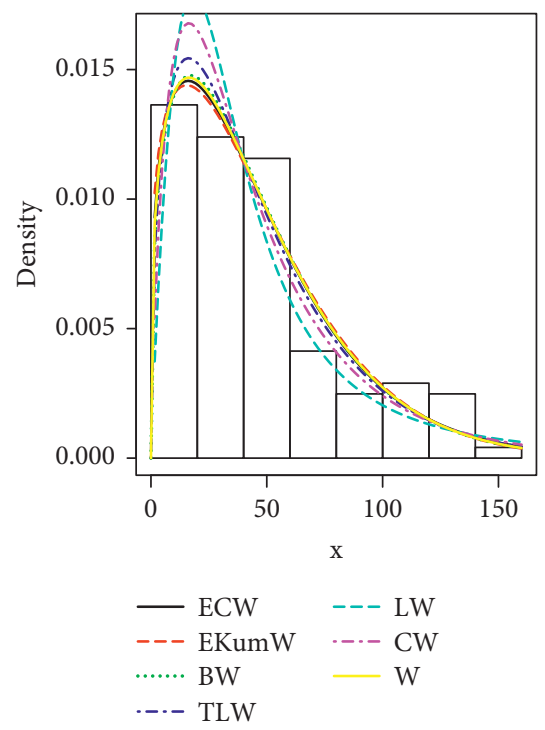

(a)

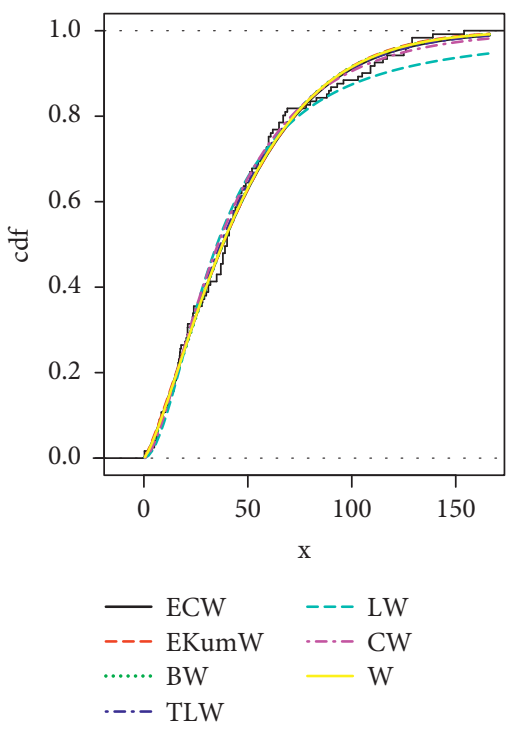

(b)

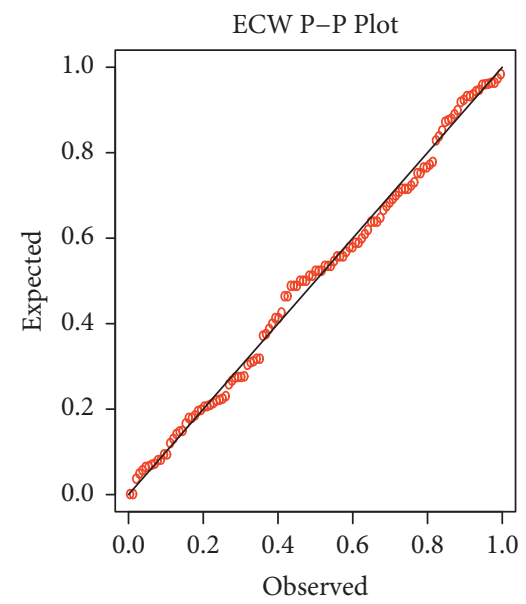

Figure 7: (a) Plots of the estimated pdfs. (b) Plots of the estimated cdfs. (c) P-P plots for cancer patients' survival times data.

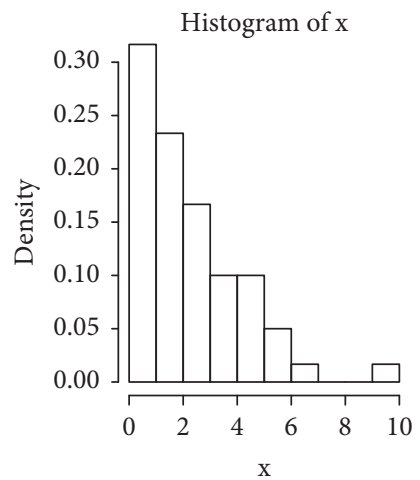

(a)

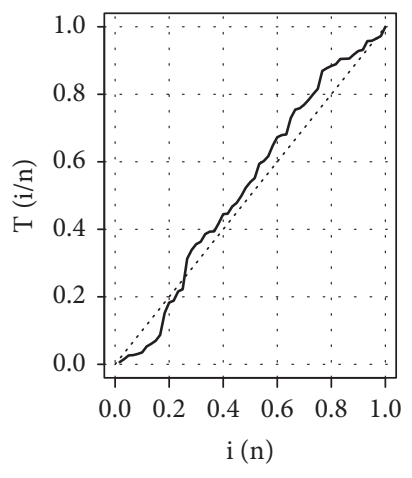

(b)

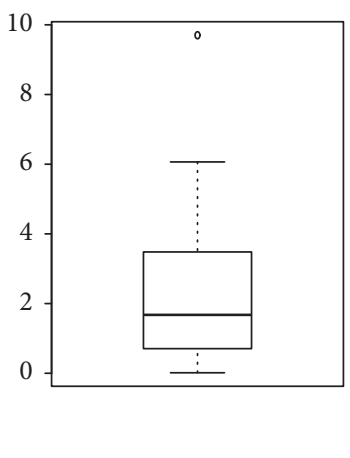

(c)

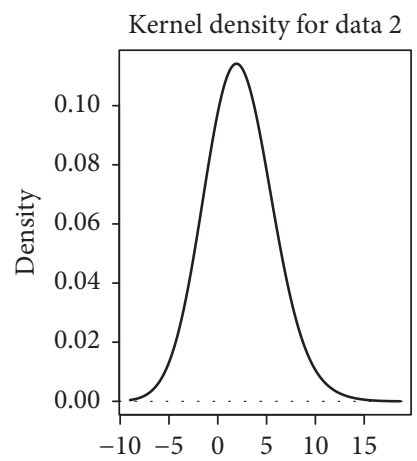

(d)

FIgUre 8: (a) Histogram. (b) TTT plot. (c) Boxplot. (d) Kernel density for electrical appliance failure data.

TABLE 11: MLEs and their standard errors (in parentheses) for electrical appliance failure data.

\begin{tabular}{|c|c|c|c|c|c|}
\hline Distribution & $\alpha$ & $\beta$ & $\lambda$ & & \\
\hline ECW & $\begin{array}{c}0.0522 \\
(0.0135)\end{array}$ & $\begin{array}{c}1.9926 \\
(0.0799)\end{array}$ & $\begin{array}{c}1.3127 \\
(0.0659)\end{array}$ & $\begin{array}{c}0.1394 \\
(0.0222)\end{array}$ & - \\
\hline EKumW & $\begin{array}{c}0.1221 \\
(0.3401)\end{array}$ & $\begin{array}{c}1.3480 \\
(2.5176)\end{array}$ & $\begin{array}{c}2.1009 \\
(6.2991)\end{array}$ & $\begin{array}{c}7.1199 \\
(46.4116)\end{array}$ & $\begin{array}{c}0.2586 \\
(0.4249)\end{array}$ \\
\hline BW & $\begin{array}{c}0.5317 \\
(0.1131)\end{array}$ & $\begin{array}{c}0.1533 \\
(0.0217)\end{array}$ & $\begin{array}{c}1.5634 \\
(0.0026)\end{array}$ & $\begin{array}{c}1.3847 \\
(0.0026)\end{array}$ & $\begin{array}{l}- \\
-\end{array}$ \\
\hline TLW & $\begin{array}{c}0.0310 \\
(0.0399)\end{array}$ & $\begin{array}{c}1.9532 \\
(0.6199)\end{array}$ & $\begin{array}{c}0.3733 \\
(0.1616)\end{array}$ & $\begin{array}{l}- \\
-\end{array}$ & - \\
\hline LW & $\begin{array}{c}0.5680 \\
(27.2443)\end{array}$ & $\begin{array}{c}0.8112 \\
(8.1398)\end{array}$ & $\begin{array}{c}2.2742 \\
(109.0685)\end{array}$ & - & - \\
\hline $\mathrm{CW}$ & $\begin{array}{c}0.8857 \\
(0.1025)\end{array}$ & $\begin{array}{c}0.6611 \\
(0.0682)\end{array}$ & $\begin{array}{l}- \\
-\end{array}$ & - & - \\
\hline W & $\begin{array}{c}0.0455 \\
(0.0814)\end{array}$ & $\begin{array}{c}1.0008 \\
(0.1066)\end{array}$ & - & - & - \\
\hline
\end{tabular}


Table 12: The statistics $\hat{\ell}, A^{*}, W^{*}, \mathrm{~K}-\mathrm{S}$, and $P$-value for electrical appliance failure data.

\begin{tabular}{|c|c|c|c|c|c|}
\hline Distribution & $\widehat{\ell}$ & $A^{*}$ & $W^{*}$ & $\mathrm{~K}-\mathrm{S}$ & $P$-value \\
\hline ECW & 105.0628 & 0.2808 & 0.0331 & 0.0614 & 0.9670 \\
\hline EKumW & 105.1225 & 0.2854 & 0.0337 & 0.0623 & 0.9622 \\
\hline BW & 105.7275 & 0.2815 & 0.0333 & 0.0676 & 0.9288 \\
\hline TLW & 105.1588 & 0.2896 & 0.0342 & 0.0626 & 0.9607 \\
\hline LW & 115.1991 & 2.0359 & 0.3285 & 0.1188 & 0.3380 \\
\hline $\mathrm{CW}$ & 110.3765 & 1.3410 & 0.2104 & 0.1041 & 0.5010 \\
\hline $\mathrm{W}$ & 107.1153 & 0.7154 & 0.1036 & 0.0777 & 0.8342 \\
\hline
\end{tabular}

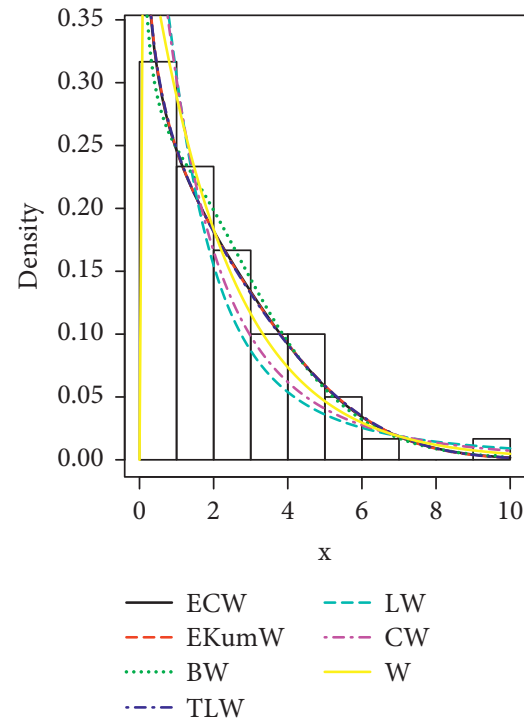

(a)

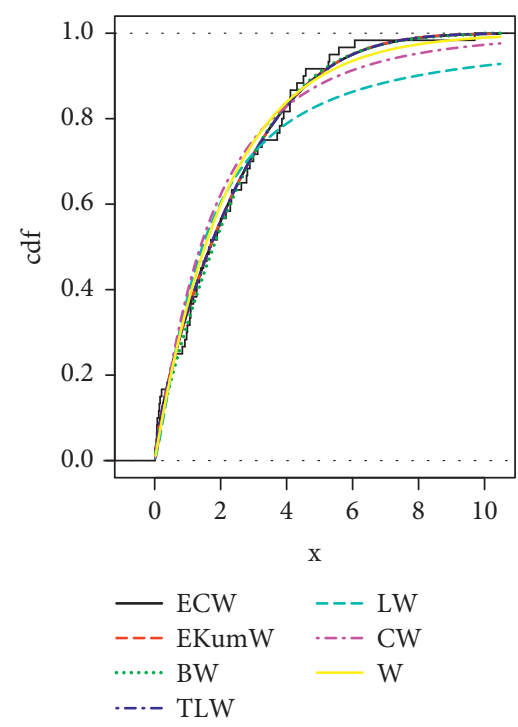

(b)

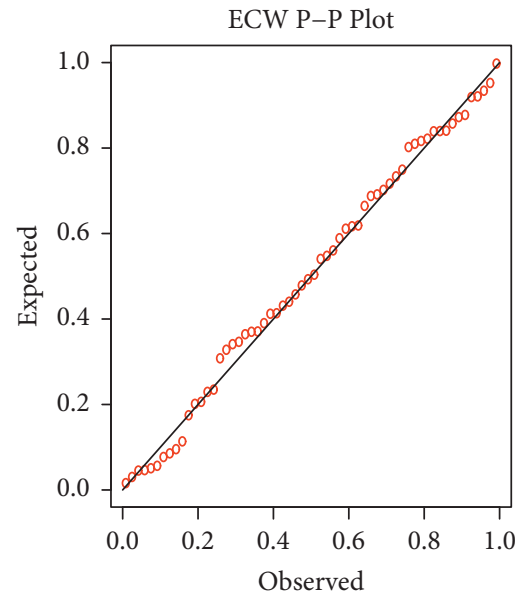

(c)

Figure 9: (a) Plots of the estimated pdfs. (b) Plots of the estimated cdfs. (c) P-P plots for electrical appliance failure data.

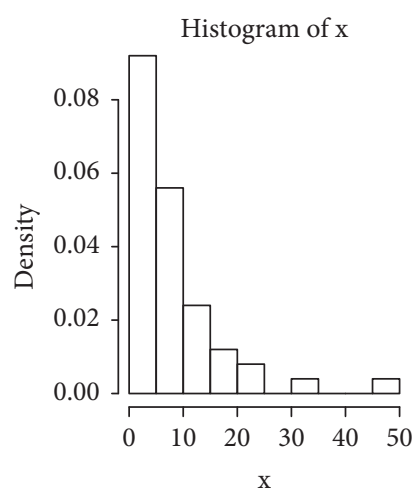

(a)

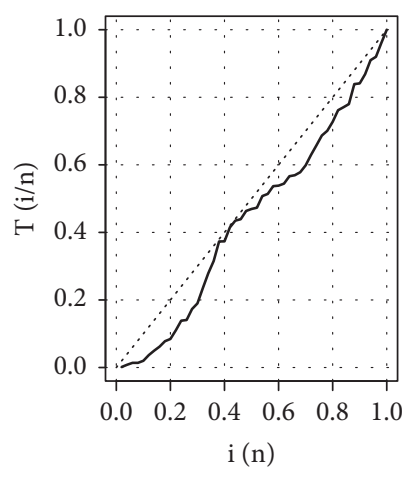

(b)

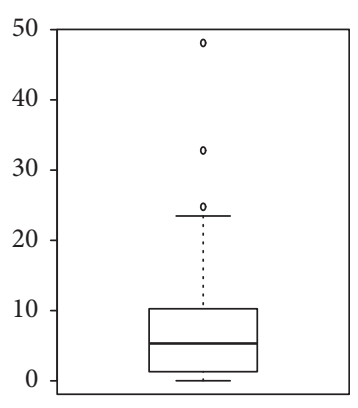

(c)

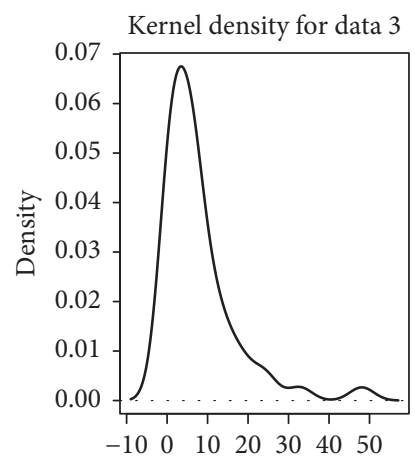

(d)

Figure 10: (a) Histogram. (b) TTT plot. (c) Boxplot. (d) Kernel density for failure data of 50 components. 
TABLE 13: MLEs and their standard errors (in parentheses) for failure data of 50 components.

\begin{tabular}{|c|c|c|c|c|c|}
\hline Distribution & $\alpha$ & $\beta$ & $\lambda$ & & \\
\hline \multirow[t]{2}{*}{ ECW } & 0.0553 & 1.1387 & 1.3788 & 0.2074 & - \\
\hline & $(0.0890)$ & $(0.4335)$ & $(2.6956)$ & $(0.3710)$ & - \\
\hline \multirow[t]{2}{*}{ EKumW } & 0.0591 & 0.5335 & 4.8871 & 8.0985 & 0.2767 \\
\hline & $(0.3013)$ & $(2.6664)$ & $(38.3064)$ & $(81.7241)$ & $(0.5569)$ \\
\hline \multirow[t]{2}{*}{ BW } & 0.5599 & 3.4542 & 0.0141 & 1.1650 & - \\
\hline & $(0.3019)$ & (7.5579) & $(0.0263)$ & $(0.4267)$ & - \\
\hline \multirow[t]{2}{*}{ TLW } & 0.6020 & 0.0349 & 1.0962 & - & - \\
\hline & $(0.3089)$ & $(0.0481)$ & $(0.3747)$ & - & - \\
\hline \multirow[t]{2}{*}{ LW } & 0.7886 & 1.3749 & 0.1443 & - & - \\
\hline & $(0.1022)$ & $(0.0635)$ & $(0.0343)$ & - & - \\
\hline \multirow[t]{2}{*}{$\mathrm{CW}$} & 0.5189 & 0.5439 & - & - & - \\
\hline & $(0.0851)$ & $(0.0603)$ & - & - & - \\
\hline \multirow[t]{2}{*}{$\mathrm{W}$} & 0.2114 & 0.8002 & - & - & - \\
\hline & $(0.0554)$ & $(0.0906)$ & - & - & - \\
\hline
\end{tabular}

Table 14: The statistics $\hat{\ell}, A^{*}, W^{*}, \mathrm{~K}-\mathrm{S}$, and $P$-value for failure data of 50 components.

\begin{tabular}{|c|c|c|c|c|c|}
\hline Distribution & $\hat{\ell}$ & $A^{*}$ & $W^{*}$ & $\mathrm{~K}-\mathrm{S}$ & $P$-value \\
\hline ECW & 150.1672 & 0.2893 & 0.0568 & 0.0948 & 0.7594 \\
\hline EKumW & 150.2794 & 0.3221 & 0.0641 & 0.1015 & 0.6810 \\
\hline BW & 150.2396 & 0.2991 & 0.0587 & 0.0956 & 0.7504 \\
\hline TLW & 150.2594 & 0.3051 & 0.0600 & 0.0967 & 0.7371 \\
\hline LW & 155.9976 & 1.3822 & 0.2573 & 0.1325 & 0.3433 \\
\hline CW & 152.5521 & 0.8232 & 0.1593 & 0.1404 & 0.2772 \\
\hline $\mathrm{W}$ & 150.6768 & 0.4272 & 0.0856 & 0.1118 & 0.5591 \\
\hline
\end{tabular}

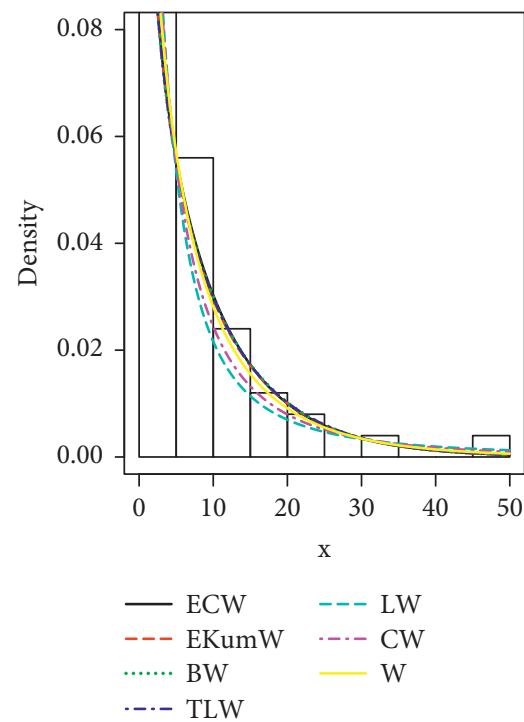

(a)

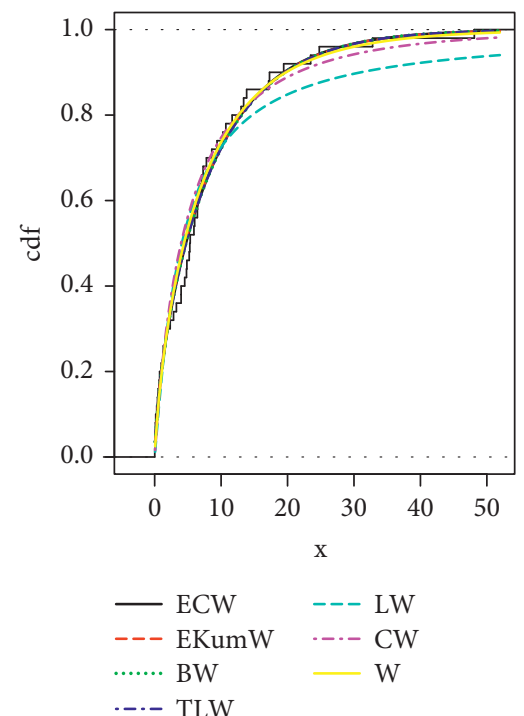

(b)

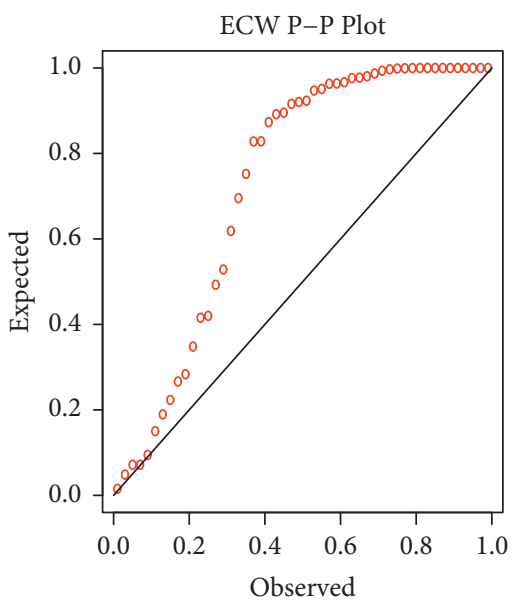

(c)

Figure 11: (a) Plots of the estimated pdfs. (b) Plots of the estimated cdfs. (c) P-P plots for failure data of 50 components.

4.3. Second Application: Electrical Appliance Failure Data. The second data presented by Lawless (2002) regarding the number of $1000 \mathrm{~s}$ of cycles to failure for 60 electrical appliances in a life test and the values are as follows:

$0.014,0.034,0.059,0.061,0.069,0.080,0.123,0.142,0.165$, $0.210,0.381,0.464,0.479,0.556,0.574,0.839,0.917,0.969$, $0.991,1.064,1.088,1.091,1.174,1.270,1.275,1.355,1.397$, $1.477,1.578,1.649,1.702,1.893,1.932,2.001,2.161,2.292$, $2.326,2.337,2.628,2.785,2.811,2.886,2.993,3.122,3.248$,
$3.715,3.790,3.857,3.912,4.100,4.106,4.116,4.315,4.510$, 4.580, 5.267, 5.299, 5.583, 6.065, and 9.701.

The summary statistics are as follows: $\min . x=0.014$, $\max \cdot x=9.7010, \quad Q 1=0.7728, \quad$ median $=1.6755$, mean $=2.1930, \quad Q 3=3.3647, \quad \mathrm{SD}=1.92, \quad$ range $=9.69$, skewness $=1.23$, and kurtosis $=2.05$.

Figure 8(a) presents the histogram of second data, showing a right tail, while the TTT plot is shown in Figure $8(\mathrm{~b})$, which is first convex and then concave. The 
boxplot is displayed in Figure 8(c), while the kernel density is shown in Figure 8(d). So, the second data is right-skewed. These graphics motivate the use of a model like ECW with a right tail.

Table 11 lists the MLEs and their corresponding standard errors (in parentheses) of the model parameters for electrical appliance failure data.

Table 12 lists the statistics $\hat{\ell}, A^{*}, W^{*}, \mathrm{~K}-\mathrm{S}$, and $P$-value for electrical appliance failure data.

Figure 9(a) presents the plots of the estimated densities, Figure 9(b) shows the plots of the estimated distribution functions, and Figure 9(c) provides the P-P plots for electrical appliance failure data.

4.4. Third Application: Failure Data of 50 Components. The third data are an uncensored dataset from Murty, Xie, and Jiang (2004) used in the industry, representing the failure time (in weeks) of 50 components put into use at time. The data values are as follows:

$0.013,0.065,0.111,0.111,0.163,0.309,0.426,0.535$, $0.684,0.747,0.997,1.284,1.304,1.647,1.829,2.336,2.838$, $3.269,3.977,3.981,4.520,4.789,4.849,5.202,5.291,5.349$, 5.911, 6.018, 6.427, 6.456, 6.572, 7.023, 7.087, 7.291, 7.787, 8.596, 9.388, 10.261, 10.713, 11.658, 13.006, 13.388, 13.842, $17.152,17.283,19.418,23.471,24.777,32.795$, and 48.105 .

The summary statistics are as follows: $\min . x=0.013$, $\max \cdot x=48.105, Q 1=1.390$, median $=5.320$, mean $=7.821$, $\mathrm{Q} 3=10.04, \mathrm{SD}=7.82$, range $=48.09$, skewness $=2.24$, and kurtosis $=6.04$.

Figure 10 (a) presents the histogram of the third dataset, showing a right tail, while the TTT plot is shown in Figure 10(b), which is convex only. The boxplot is displayed in Figure 10(c), while the kernel density is shown in Figure $10(\mathrm{~d})$, and the conclusion is that the third dataset is right-skewed. These graphics motivate the use of a model with a right tail, such as the ECW model.

Table 13 lists the MLEs and their corresponding standard errors (in parentheses) of the model parameters for failure data of 50 components.

Table 14 lists the statistics $\widehat{\ell}, A^{*}, W^{*}, \mathrm{~K}-\mathrm{S}$, and $P$-value for failure data of 50 components.

Figure 11(a) presents the plots of the estimated densities, Figure 11(b) shows the plots of the estimated distribution functions, and Figure 11(c) provides the P-P plots for failure data of 50 components.

\section{Concluding Remarks}

An extended version of the cosine-G family of distributions is introduced, utilizing parameter induction methodology and trigonometric generalizers concurrently. This family is proved to be more flexible and better fitting in many practical situations, especially survival and reliability modeling. Moreover, a four-parameter model (ECW) is derived with symmetrical, right-skewed, left-skewed, reversed-J, and bimodal reversed-J density shapes and increasing, decreasing, bathtub, and upsidedown bathtub hazard rate shapes. Several mathematical, numerical, and structural properties such as linear representation for cdf and pdf, moments and weighted moments, quantile function, order statistics, entropies, and stochastic ordering are also derived.

The parametric estimation is exercised via the maximum likelihood method, and the adequacy of the estimates is attested by the Monte Carlo simulation study. We use a variety of well-known goodness-of-fit statistics to test which distribution is best suited to survival and failure datasets. We conclude that this proposed distribution consistently provides better fits than other existing and competing models even with more number of parameters. We hope that the proposed family and derived models will draw broader applications in a number of areas such as reliability and survival research, hydrology, geology, and several other fields [44-50].

\section{Data Availability}

All data are available in the paper.

\section{Conflicts of Interest}

The authors declare that they have no conflicts of interest.

\section{Acknowledgments}

This study was supported by Taif University Researchers Supporting Project (no. TURSP-2020/318), Taif University, Taif, Saudi Arabia.

\section{References}

[1] L. Souza, New Trigonometric Classes of Probabilistic Distributions, Thesis, Universidade Federal Rural de Pernambuco, Brazil, 2015.

[2] H. M. Alshanbari, A. A. H. Abd El-Bagoury, A. M. Gemeay, E. H. Hafez, and A. S. Eldeeb, "A flexible extension of pareto distribution," Properties and Applications Computational Intelligence and Neuroscience, vol. 2021, Article ID 9819200, 17 pages, 2021.

[3] A.-E. A. M. Teamah, A. Elbanna, and A. M. Gemeay, "Heavytailed log-logistic distribution: properties, risk measures and applications," Statistics, Optimization \& Information Computing, vol. 9, no. 4, pp. 910-941, 2021.

[4] X. Liu, Z. Ahmad, A. M. Gemeay, A. T. Abdulrahman, E. H. Hafez, and N. Khalil, "Modeling the survival times of the COVID-19 patients with a new statistical model: a case study from China," PLoS One, vol. 16, no. 7, Article ID e0254999, 2021.

[5] A. Alzaatreh, C. Lee, and F. Famoye, "A new method for generating families of continuous distributions," Metron, vol. 71, no. 1, pp. 63-79, 2013.

[6] C. Alexander, G. M. Cordeiro, E. M. M. Ortega, and J. M. Sarabia, "Generalized beta-generated distributions," Computational Statistics \& Data Analysis, vol. 56, no. 6, pp. 1880-1897, 2012.

[7] G. M. Cordeiro, E. M. M. Ortega, and D. C. C. Da Cunha, "The exponentiated generalized class of distributions," Journal of Data Science, vol. 11, pp. 1-27, 2013.

[8] D. H. Raab and E. H. Green, "A cosine approximation to the normal distribution," Psychometrika, vol. 26, no. 4, pp. 447-450, 1961. 
[9] S. Nadarajah and S. Kotz, "Beta trigonometric distributions," Portuguese Economic Journal, vol. 5, no. 3, pp. 207-224, 2006.

[10] S. Chakraborty, PJ. Hazarika, and MM. Ali, "A new skew logistic distribution and its properties," Pakistan Journal of Statistics, vol. 28, no. 4, pp. 513-524, 2012.

[11] C. Chesneau, H. S. Bakouch, and T. Hussain, "A new class of probability distributions via cosine and sine functions with applications," Communications in Statistics - Simulation and Computation, vol. 48, no. 8, pp. 2287-3000, 2019.

[12] L. Souza, W. R. O. Junior, C. C. R. De Brito, C. Chesneau, T. A. E. Ferreira, and L. Soares, "General properties for the Cos-G class of distributions with applications," Eurasian Bulletin of Mathematics, vol. 2, no. 2, pp. 63-79, 2019.

[13] Z. Mahmood, C. Chesneau, and M. H. Tahir, "A new sine-G family of distributions: properties and applications," Bulletin of Computational Applied Mathematics, vol. 7, no. 1, pp. 53-81, 2019.

[14] C. Chesneau, H. S. Bakouch, T. Hussain, and B. Para, "The cosine geometric distribution with count data modeling," Journal of Applied Statistics, vol. 48, no. 1, pp. 124-137, 2021.

[15] C. Chesneau and F. Jamal, "The sine Kumaraswamy-G family of distributions," Journal of Mathematical Extension, vol. 15, 2020.

[16] A. A. Al-Babtain, I. Elbatal, C. Chesneau, and M. Elgarhy, "Sine Topp-Leone-G family of distributions: theory and applications," Open Physics, vol. 18, no. 1, pp. 574-593, 2020.

[17] A. Ali, "Sine power lomax model with application to bladder cancer data," Nanoscience and NanoTechnology Letters, vol. 12, no. 5, pp. 677-684, 2020.

[18] I. Alkhairy, M. Nagy, A. Hassan Muse, and E. Hussam, "The arctan-X family of distributions: properties, simulation, and applications to actuarial sciences," Complexity, vol. 2021, Article ID 4689010, 14 pages, 2021.

[19] V. B. V. Nagarjuna, R. V. Vardhan, and C. Chesneau, "On the accuracy of the sine power lomax model for data fitting," Modelling, vol. 2, pp. 78-104, 2020.

[20] V. B. V. Nagarjuna, R. V. Vardhan, and C. Chesneau, "Kumaraswamy generalized power Lomax distribution," Stat, vol. 4, pp. 28-45, 2021.

[21] T. R. Ahmed, H. T. Ahlam, and S. E. D. Beih, "Generalized power akshaya distribution and its applications open," Journal of Modelling and Simulation, vol. 9, no. 4, pp. 323-338, 2021.

[22] H. M. Abdisalam, H. T. Ahlam, F. Eman et al., "Modelling the COVID-19 mortality rate with a new versatile modification of the log-logistic distribution," Computational Intelligence and Neuroscience, vol. 2021, Article ID 8640794, 14 pages, 2021.

[23] E. M. Almetwally, "The odd Weibull inverse topp-leone distribution with applications to COVID-19 data," Annals of Data Science, vol. 1, 2021.

[24] A. Hassan, E. M. Almetwally, and G. M. Ibrahim, "Kumaraswamy inverted t-leone distribution with applications to COVID-19 data," Computers, Materials \& Continua, vol. 68, no. 1, pp. 337-358, 2021.

[25] E. M. Almetwally, M. A. Sabry, R. Alharbi, D. Alnagar, S. A. Mubarak, and E. H. Hafez, "Marshall-olkin alpha power Weibull distribution: different methods of estimation based on type-I and type-II censoring," Complexity, vol. 2021, Article ID 5533799, 18 pages, 2021.

[26] E. M. Almetwally and H. A. Haj Ahmad, "A new generalization of the Pareto distribution and its applications," Statistics in Transition New Series, vol. 21, no. 5, pp. 61-84, 2020.

[27] H. M. Almongy, E. M. Almetwally, and A. E. Mubarak, "Marshall-olkin alpha power lomax distribution: estimation methods, applications on physics and economics," Pakistan
Journal of Statistics and Operation Research, pp. 137-153, 2021.

[28] H. M. Almongy, E. M. Almetwally, H. M. Aljohani, A. S. Alghamdi, and E. H. Hafez, "A new extended Rayleigh distribution with applications of COVID-19 data," Results in Physics, vol. 23, Article ID 104012, 2021.

[29] A. A. Al-Babtain, I. Elbatal, H. Al-Mofleh, A. M. Gemeay, A. Z. Afify, and A. M. Sarg, "The flexible burr X-G family: properties, inference, and applications in engineering science," Symmetry, vol. 13, no. 3, p. 474, 2021.

[30] G. G. Hamedani, M. Ç. Korkmaz, N. S. Butt, and H. M. Yousof, "The type I quasi lambert family: properties, characterizations and different estimation methods," Pakistan Journal of Statistics and Operation Research, vol. 17, no. 3, pp. 545-558, 2021.

[31] E. Altun, M. C. Korkmaz, M. El-Morshedy, and M. S. Eliwa, "A new flexible family of continuous distributions: the additive odd-G family," Mathematics, vol. 9, no. 16, 2021.

[32] M. A. Aljarrah, C. Lee, and F. Famoye, "On generating T-X family of distributions using quantile functions," Journal of Statistical Distributions and Applications, vol. 1, no. 2, 2014.

[33] N. Salahuddin, A. Khalil, W. K. Mashwani, H. Shah, P. Jomsri, and T. Panityakul, "A novel generalized family of distributions for engineering and life sciences data applications," Meta-Heuristic Techniques for Solving Computational Engineering Problems, vol. 2021, Article ID 9949999, 16 pages, 2021.

[34] M. Ç. Korkmaz, E. Altun, H. M. Yousof, and G. G. Hamedani, "The $\mathrm{h}$ generator of distributions: properties, characterizations, regression modeling and applications," Journal of Statistical Theory and Applications, vol. 19, no. 1, pp. 59-74, 2020.

[35] M. Cordeiro, A. Emrah, M. Korkmaz, and M. Haitham, "The XGamma family: censored regression modelling and applications," Revstat - Statistical Journal, vol. 18, no. 5, pp. 593-612, 2020.

[36] M. Ç. Korkmaz, H. M. Yousof, M. Alizadeh, and G. G. Hamedani, "The Topp-Leone generalized odd log-logistic family of distributions: properties, Characterizations and Applications," Communications Faculty Of Science University of Ankara Series A1Mathematics and Statistics, vol. 68, no. 2, pp. 1506-1527, 2019.

[37] F. A. Bhatti, M. C. Korkmaz, G. G. Hamedani, G. M. Cordeiro, and M. Ahmad, "On Burr III Marshal Olkin family: development, properties, characterizations and applications," Journal of Statistical Distributions and Applications, vol. 6, no. 12, pp. 45-72, 2019.

[38] M. A. Alizadeh, M. Ç. Korkmaz, J. A. Almamy, and A. A. E. Ahmed, "Another odd log-logistic logarithmic class of continuous distributions," Journal of Statisticians: Statistics and Actuarial Sciences, vol. 2, pp. 55-72, 2021.

[39] A. Algarni, A. M Almarashi, I. Elbatal et al., "Type I half logistic Burr XG family: properties, bayesian, and nonbayesian estimation under censored samples and applications to COVID-19 data," Mathematical Problems in Engineering, vol. 2021, Article ID 5461130, 21 pages, 2021.

[40] M. Shaked and J. G. Shanthikumar, Stochastic Orders and Their Applications, Academic Press, New York, NY, USA, 1994.

[41] H. A. David and H. N. Nagaraja, Order Statistics, John Wiley \& Sons, New Jersey, NY, USA, 2003.

[42] G. Chen and N. Balakrishnan, "A general purpose approximate goodness-of-fit test," Journal of Quality Technology, vol. 27, no. 2, pp. 154-161, 1995. 
[43] E. T. Lee, Statistical Methods for Survival Data Analysis, John Wiley, New York, NY, USA, 1992.

[44] N. Eugene, C. Lee, and F. Famoye, "Beta-normal distribution and its applications," Communications in Statistics - Theory and Methods, vol. 31, no. 4, pp. 497-512, 2002.

[45] A. Z. Keller and A. R. Kamath, "Alternative reliability models for mechanical systems," in Proceedings of the Third International Conference on Reliability and Maintainability, pp. 411-415, Toulouse, France, 1982.

[46] JF. Kenney and E. S. Keeping, Mathematics of Statistics, Chapman \& Hall, New Jersey, NY, USA, 3 edition, 1962.

[47] M. Ç. Korkmaz, "A new family of the continuous distributions: the extended Weibull-G family," Communications Faculty Of Science University of Ankara Series A1Mathematics and Statistics, vol. 68, no. 1, pp. 248-270, 2018.

[48] D. Kumar, U. Singh, and S. K. Singh, "A new distribution using sine function- its application to bladder cancer patients," Journal of Statistics Applications and Probability, vol. 4, no. 3, pp. 417-427, 2015.

[49] J. J. A. Moors, "A quantile alternative for kurtosis," The Statistician, vol. 37, no. 1, pp. 25-32, 1988.

[50] R Development Core Team, A Languge and Environment for Statistical Computing, R Foundation for Statistical Computing, Austria, Vienna, 2009. 